\title{
The molecular gas content of the Pipe Nebula
}

\section{Direct evidence of outflow-generated turbulence in B59?}

\author{
A. Duarte-Cabral ${ }^{1,2,3}$, A. Chrysostomou ${ }^{4}$, N. Peretto ${ }^{5}$, G. A. Fuller ${ }^{3}$, B. Matthews ${ }^{6,7}$, G. Schieven ${ }^{6,7}$, and G. R. Davis ${ }^{4}$ \\ 1 Univ. Bordeaux, LAB, UMR 5804, 33270 Floirac, France \\ e-mail: Ana.Cabral@obs.u-bordeaux1.fr \\ 2 CNRS, LAB, UMR 5804, 33270 Floirac, France \\ 3 Jodrell Bank Centre for Astrophysics, School of Physics and Astronomy, University of Manchester, Oxford Road, Manchester, \\ M13 9PL, UK \\ 4 Joint Astronomy Centre, 660 North Aohoku Place, Hilo, HI 96720, USA \\ 5 Laboratoire AIM, CEA/DSM-CNRS-Université Paris Diderot, IRFU/Service d'Astrophysique, C.E. Saclay, Orme de merisiers, \\ 91191 Gif-sur-Yvette, France \\ ${ }^{6}$ Herzberg Institute of Astrophysics, National Research Council Canada, 5071 West Saanich Road., Victoria, BC, V9E 2E7, Canada \\ 7 University of Victoria, Finnerty Road, Victoria, BC, V8W 3P6, Canada
}

Received 18 March 2012 / Accepted 12 May 2012

\section{ABSTRACT}

\begin{abstract}
Context. Star forming regions may share many characteristics, but the specific interplay between gravity, magnetic fields, large-scale dynamics, and protostellar feedback will have an impact on the star formation history of each region. The importance of feedback from outflows is a particular subject to debate, as we are yet to understand the details of their impact on clouds and star formation.

Aims. The Pipe Nebula is a nearby molecular cloud hosting the B59 region as its only active star-forming clump. This paper focuses on the global dynamics of B59, its temperature structure, and its outflowing gas, with the goal of revealing the local and global impact of the protostellar outflows.

Methods. Using HARP at the James Clerk Maxwell Telescope, we have mapped the B59 region in the $J=3 \rightarrow 2$ transition of ${ }^{12} \mathrm{CO}$ to study the kinematics and energetics of the outflows, and the same transitions of ${ }^{13} \mathrm{CO}$ and $\mathrm{C}^{18} \mathrm{O}$ to study the overall dynamics of the ambient cloud, the physical properties of the gas, and the hierarchical structure of the region.

Results. The B59 region has a total of $\sim 30 M_{\odot}$ of cold and quiescent material, mostly gravitationally bound, with narrow line widths throughout. Such low levels of turbulence in the non-star-forming regions within B59 are indicative of the intrinsic initial conditions of the cloud. On the other hand, close to the protostars the impact of the outflows is observed as a localised increase of both $\mathrm{C}^{18} \mathrm{O}$ line widths from $\sim 0.3 \mathrm{~km} \mathrm{~s}^{-1}$ to $\sim 1 \mathrm{~km} \mathrm{~s}^{-1}$, and ${ }^{13} \mathrm{CO}$ excitation temperatures by $\sim 2-3 \mathrm{~K}$. The impact of the outflows is also evident in the low column density material which shows signs of being shaped by the outflow bow shocks as they pierce their way out of the cloud. Much of this structure is readily apparent in a dendrogram analysis of the cloud and demonstrates that when decomposing clouds using such techniques a careful interpretation of the results is needed.

Conclusions. The low mass of B59 together with its intrinsically quiescent gas and small number of protostars, allows the identification of specific regions where the outflows from the embedded sources interact the dense gas. Our study suggests that outflows are an important mechanism for injecting and sustaining supersonic turbulence at sub-parsec size scales. We find that less than half of the outflow energy is deposited as turbulent energy of the gas, however this turbulent energy is sufficient to slow down the collapse of the region.
\end{abstract}

Key words. stars: formation - stars: protostars - ISM: clouds - ISM: jets and outflows - ISM: kinematics and dynamics ISM: individual objects: B59

\section{Introduction}

As stellar nurseries, molecular clouds contain the ingredients for star formation. However, the physical processes which prevent or trigger star formation have been the subject of debate over the past decade (e.g. McKee \& Ostriker 2007; Vázquez-Semadeni 2010; Hennebelle \& Chabrier 2011). The relative importance of the various processes is unlikely to be universal: while in some regions dynamical effects such as a collision of clouds or convergence of flows (e.g. Vázquez-Semadeni et al. 2007; Schneider et al. 2010; Duarte-Cabral et al. 2011) may be significant, other regions can evolve more quietly, from quiescent material possibly controlled by the local magnetic field (e.g. Nakamura \& Li 2008; Alves et al. 2008). The imprints of the initial conditions specific to a given cloud are potentially preserved in the properties of the gas and dust of their young proto-clusters although once star formation begins feedback from the forming stars can mask the signatures of these initial conditions.

Young protostellar outflows may be responsible for clearing and disrupting protostellar envelopes, limiting the reservoir of mass which will end up being accreted by the central protostars (e.g. Fuller \& Ladd 2002; Arce \& Sargent 2006; Arce et al. 2010). Even though an outflow may carry sufficient energy to unbind all of an envelope, this energy may well be deposited far from the dense gas, or even outside the molecular cloud. Knowing exactly where the outflow momentum and energy are deposited is fundamental for understanding whether outflows are a source of turbulence capable of providing support against gravity, clearing circumstellar regions and slowing (or enhancing) star formation. However, the efficiency with which outflows can 
drive turbulence is strongly debated (see review from McKee $\&$ Ostriker 2007). The analytical model of Matzner \& McKee (2000) estimates a typical outflow injection scale, the scale at which the momentum is most efficiently deposited, of a few tenths of a parsec. Numerical models (e.g. Nakamura \& Li 2007; Carroll et al. 2009, 2010) confirm that outflows can drive supersonic turbulence at sub-outflow scales and produce smooth velocity structures on outflow-scales that sweep up and dissipate smaller structures, even though outflows are not the primary source of turbulence at all scales in molecular clouds. The effect of the outflows is to produce an overall flatter density spectrum, which limits the fragmentation and infall of material onto the final protostars.

Observationally, the evidence suggests that outflows can provide barely enough kinetic energy to support entire clouds against gravity (e.g. Arce et al. 2010; Maury et al. 2009), even though they may be able to maintain the supersonic turbulence at sub-parsec scales (e.g. Nakamura et al. 2011).

Nevertheless, the importance of outflows on the energy balance of a region is likely to depend on the physical properties of the region. However, it is observationally difficult to measure the direct injection of turbulence due to outflows and assess its impact on star formation. One reason is that in dynamic clusterforming regions, with large-scale motions, and on-going star formation, the direct effect of outflows can be easily confused and masked by other dynamical events.

Here we present a study of the B59 star-forming region, within the generally quiescent Pipe Nebula. Low-density clouds such as the Pipe provide the best opportunities to directly observe the impact of outflows where stars have began to form. In Sect. 2 we present an overview of the Pipe Nebula and its active region, B59. In Sect. 3 we detail the observations, and we present the results on the physical properties in Sect. 4, including optical depth and estimates of excitation temperatures. The outflows of the region are presented in Sect. 5. The ambient cloud's dynamics and hierarchical structure are described in Sect. 6 (the details on the hierarchical structure of B59, performed using a dendrogram technique, is described in detail in Appendix A). Finally, we discuss the outflow-dense gas interaction in Sect. 7, and we outline our conclusions in Sect. 8.

\section{The Pipe Nebula and B59}

The Pipe Nebula, located at $\sim 130 \mathrm{pc}^{1}$ from the Sun (Lombardi et al. 2006), is part of the Gould Belt of star-forming regions. The global features of this cloud appear similar to other Gould Belt clouds, with a total mass comparable to the few $\times 10^{4} M_{\odot}$ of the Taurus-Auriga complex, and a filamentary structure with a magnetic field threading it (Alves et al. 2008). However the Pipe has an extremely low star formation efficiency of less than $0.1 \%$ compared to other Gould Belt clouds which have efficiencies of $\sim 2-20 \%$. In fact, only one active star forming clump, B59, has been found thus far in the Pipe cloud (Brooke et al. 2007; Forbrich et al. 2009, 2010). This small group of protostars within B59 are known to power more than one molecular outflow (Onishi et al. 1999; Riaz et al. 2009). Figure 1 shows the

\footnotetext{
1 Alves \& Franco (2007) have estimated a distance of 145 pc, but we adopt $130 \mathrm{pc}$ as it lies within the uncertainties of these authors, and it is the distance most commonly adopted in other studies of the Pipe Nebula. We note, however, that uncertainties on the distance are of the order of $10 \%$ and we are therefore possibly underestimating masses by $20 \%$ by assuming the nearer distance of $130 \mathrm{pc}$.
}

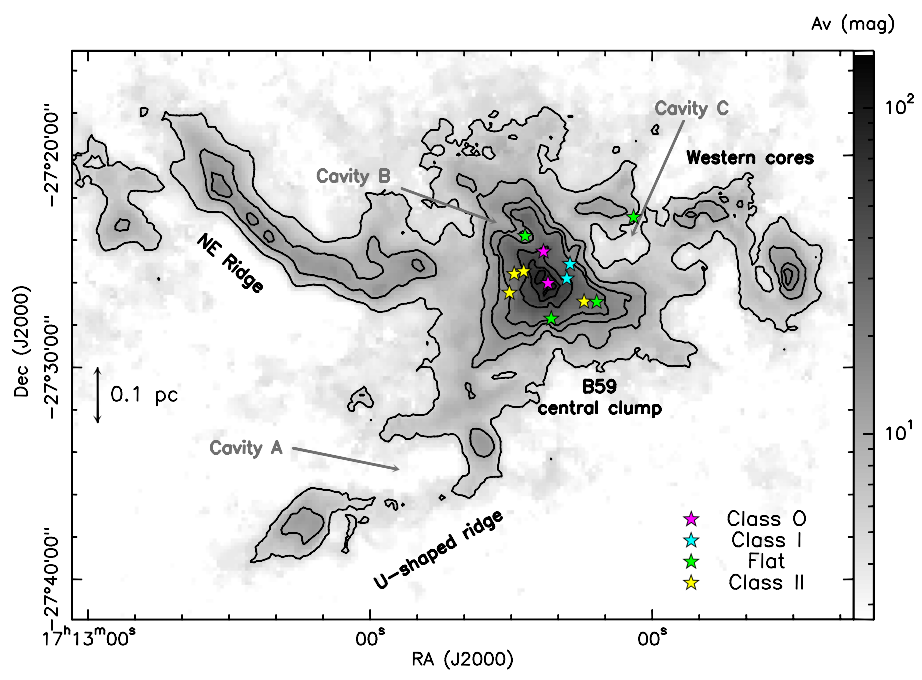

Fig. 1. Extinction map of B59 in grayscale and contours (RománZúñiga et al. 2009), showing the known young stellar objects (YSOs) in the region (from Brooke et al. 2007; Forbrich et al. 2009), except for the Class III objects. Contours range from an $A_{v}$ of 5 to 20 in steps of 5, and then by steps of 20 . Note that there are only four known Class 0 and I protostars in the region. The black labels show designations of regions within B59 as adopted throughout the paper. Some cavities are indicated with grey arrows and labels, and are discussed further in the text.

extinction map of B59 and identifies several features and regions which will be referred to in this work.

Several scenarios have been suggested for the formation of B59 as an active star-forming clump at the edge of the Pipe Nebula. One suggests that B59 formed solely governed by gravity (Heitsch et al. 2009). In another view, the magnetic field plays an important role guiding the collapse of material towards the main filament (Alves et al. 2008). Peretto et al. (2012) propose that the formation of B59 at the edge of the Pipe is due to a compression front (west of the B59, beyond the western cores) from a nearby OB association which has gathered the gas in a "snowplough" fashion. The effects of gravitational focusing playing a role later on, with a contraction of the gathered gas and the formation of stars in the centre of B59.

Our previous knowledge of the molecular gas structure in the Pipe Nebula, however, was limited to the $4^{\prime}$ resolution maps of the $J=1 \rightarrow 0$ transitions of ${ }^{12} \mathrm{CO},{ }^{13} \mathrm{CO}$ and $\mathrm{C}^{18} \mathrm{O}$ by Onishi et al. (1999). Beyond this, molecular line observations of the Pipe Nebula consist of single spectra of dense gas tracers such as $\mathrm{NH}_{3}, \mathrm{CCS}, \mathrm{HC}_{5} \mathrm{~N}$ and other early- and late-time molecules (Rathborne et al. 2008; Frau et al. 2010, 2012), and $\mathrm{C}^{18} \mathrm{O}$ (Muench et al. 2007) towards the peaks of cores identified in $1^{\prime}$ resolution extinction maps (Lombardi et al. 2006; Román-Zúñiga et al. 2010).

\section{Observations}

Using HARP at the James Clerk Maxwell Telescope (JCMT, Buckle et al. 2009) in May and June 2010, we have mapped ${ }^{13} \mathrm{CO}$ and $\mathrm{C}^{18} \mathrm{O} J=3 \rightarrow 2$ (at 330.6 and $329.3 \mathrm{GHz}$ respectively) over the entire B59 star-forming region $\left(\sim 0.11 \mathrm{deg}^{2}\right)$. The observations were taken with a sky opacity at $225 \mathrm{GHz}$ varying between 0.05 and 0.08 . The data were observed as several raster maps which have an original spatial resolution of $15^{\prime \prime}$ and a spectral resolution of $0.05 \mathrm{~km} \mathrm{~s}^{-1}$. The rms noise level on the final B59 dataset is $0.22 \mathrm{~K}$ (in $T_{\mathrm{A}}^{*}$ ) with $0.25 \mathrm{~km} \mathrm{~s}^{-1}$ 

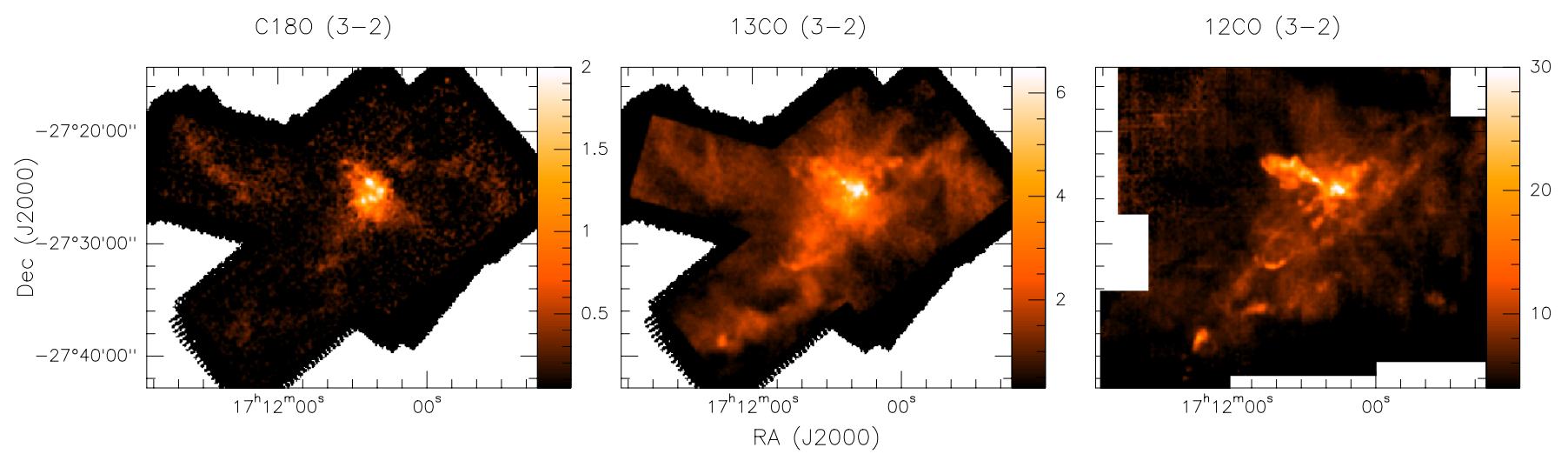

Fig. 2. Integrated intensity $\left(\int T_{\mathrm{A}}^{*} \mathrm{~d} v \mathrm{~K} \mathrm{~km} \mathrm{~s}^{-1}\right)$ maps of the three isotopologues observed. For the $\mathrm{C}^{18} \mathrm{O}$ (left) and ${ }^{13} \mathrm{CO}$ (middle), the integration range was 1 to $5.5 \mathrm{~km} \mathrm{~s}^{-1}$. The ${ }^{12} \mathrm{CO}$ (right) was integrated from -5 to $15 \mathrm{~km} \mathrm{~s}^{-1}$. Here we can see that the $\mathrm{C}^{18} \mathrm{O}$ follows the shape as seen in extinction (Fig. 1), while the ${ }^{13} \mathrm{CO}$ emission starts to pick up the outflowing material, best seen in the ${ }^{12} \mathrm{CO}$ emission dominated by high velocity gas.

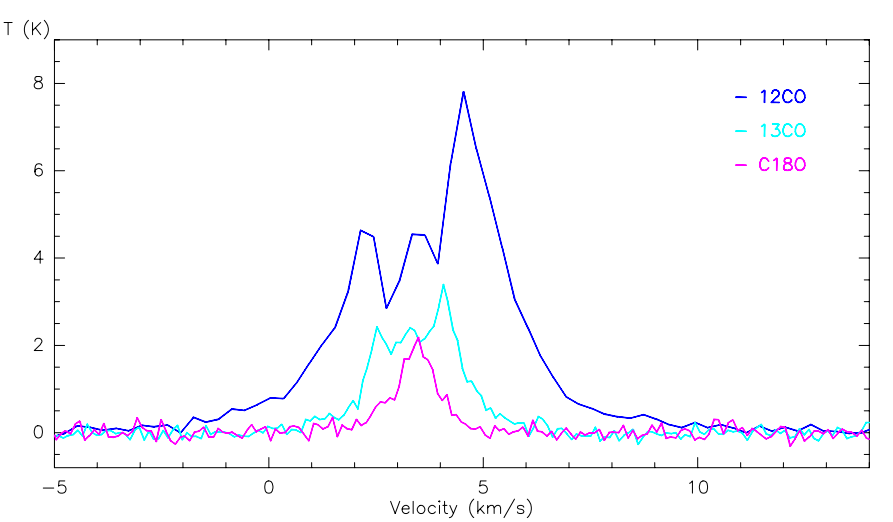

Fig. 3. Plot of $\mathrm{C}^{18} \mathrm{O}$ (purple line), ${ }^{13} \mathrm{CO}$ (light blue line), with a velocity resolution of $0.1 \mathrm{~km} \mathrm{~s}^{-1}$, and ${ }^{12} \mathrm{CO}$ (dark blue line), with a velocity resolution of $0.3 \mathrm{~km} \mathrm{~s}^{-1}$, in B59, at the position of the Class 0 source B11, at $\mathrm{RA}=17^{\mathrm{h}} 11^{\mathrm{m}} 23.0^{\mathrm{s}}$, Dec $=-27^{\circ} 24^{\prime} 32.8^{\prime \prime}$, showing the outflow wings and self absorption dip in ${ }^{13} \mathrm{CO}$ and ${ }^{12} \mathrm{CO}$.

channels. Complementary ${ }^{12} \mathrm{CO} J=3 \rightarrow 2(345.8 \mathrm{GHz})$ data were obtained with HARP using JCMT Director's discretionary time. These data reached an rms noise level of $0.2 \mathrm{~K}$ (in $T_{\mathrm{A}}^{*}$ ) at $0.5 \mathrm{~km} \mathrm{~s}^{-1}$ velocity resolution. All data were corrected for the telescope main beam efficiency of $\eta_{\mathrm{mb}}=0.66$ (Buckle et al. 2009; Curtis et al. 2010a).

The data reduction was performed using the ORAC-DR pipeline (Cavanagh et al. 2008), using the recipe REDUCE SCIENCE NARROWLINE, with minor modifications tailored for our dataset, with a pixel size of $7.4^{\prime \prime}$. This reduction procedure automatically fits and corrects baselines and removes bad detectors based on the time-series data and on the rms noise levels of the final maps. The final reduced maps for each area were gridded together using Starlink software.

All datacubes were convolved to a $20^{\prime \prime}$ resolution in order to suppress some of the high frequency noise, and were masked to pixels where the peak signal to noise (estimated at each pixel) was higher than 4 in the smoothed map. This masking procedure was particularly effective for the weaker $\mathrm{C}^{18} \mathrm{O}$, but it left virtually unchanged the datacubes for the other two isotopologues. The reduced integrated intensity maps are presented in Figs. 2 and 3 shows an example of spectra at the position of a protostellar source.

\section{Opacity and temperature structure of B59}

\subsection{CO-to- $\mathrm{H}_{2}$ relation}

The column density structure of B59 as seen with the dust extinction and emission is now relatively well known (Román-Zúñiga et al. 2009, 2012; Peretto et al. 2012). Unlike the dust and gas emission, the dust extinction is not sensitive to the temperature. As such, studying the relation between the $\mathrm{H}_{2}$ column densities derived from the dust extinction and the gas emission can give an insight to optical depth effects, temperature effects and the local abundance of a molecule with respect to $\mathrm{H}_{2}$.

Figure 4 presents the relation between $\mathrm{H}_{2}$ column densities and the ${ }^{13} \mathrm{CO}$ and $\mathrm{C}^{18} \mathrm{O}$ emission in B59 as scatter plots. The $\mathrm{H}_{2}$ column density was derived from the extinction map of B59 (Román-Zúñiga et al. 2009) assuming $N\left(\mathrm{H}_{2}\right) / A_{\mathrm{v}}=9.4 \times$ $10^{20} \mathrm{~cm}^{-2} \mathrm{mag}^{-1}$ (e.g. Bohlin et al. 1978; Pineda et al. 2008). This is plotted against the ${ }^{13} \mathrm{CO}$ and $\mathrm{C}^{18} \mathrm{O}$ integrated intensities corrected for the telescope efficiency. Both the extinction and the molecular line maps have $20^{\prime \prime}$ resolution and were resampled to a common area and pixel size, to enable a pixel-by-pixel comparison.

In the figure, the coloured symbols indicate the correlation predicted by the non-LTE radiative transfer model RADEX (van der Tak et al. 2007) for different kinetic temperatures, using a volume density above the critical densities of ${ }^{13} \mathrm{CO}$ and $\mathrm{C}^{18} \mathrm{O}(3-2)$. The $\mathrm{H}_{2}$ column densities are estimated assuming a fractional abundance of ${ }^{13} \mathrm{CO}$ and $\mathrm{C}^{18} \mathrm{O}$ with respect to $\mathrm{H}_{2}$ of $1.4 \times 10^{-6}$ and $1.7 \times 10^{-7}$ respectively (Frerking et al. 1982). These models are only meant to illustrate the behaviour of the emission at different temperatures, and they do not represent actual fits to the datapoints. The curves show the turn-over between a linear correlation between gas emission and $\mathrm{H}_{2}$ column density and where the lines become optically thick. The linear portion of the curve is related to the abundance of ${ }^{13} \mathrm{CO}$ and $\mathrm{C}^{18} \mathrm{O}$ relative to $\mathrm{H}_{2}$.

To estimate the masses and column densities directly from the gas emission (Appendix A), we estimate a "conversion" factor, $X_{\mathrm{CO}}$, on the linear portion of the datapoints as $X_{\mathrm{CO}}=$ $N\left(\mathrm{H}_{2}\right) / I_{\mathrm{CO}}$ (e.g. Pineda et al. 2008), where $N\left(\mathrm{H}_{2}\right)$ is the $\mathrm{H}_{2}$ column density and $I_{\mathrm{CO}}$ is the integrated intensity of the molecular transition.

For $\mathrm{C}^{18} \mathrm{O}$, a linear fit to all the datapoints provides a $X_{\mathrm{C}^{18} \mathrm{O}}=1.82 \times 10^{22} \mathrm{~cm}^{-2} \mathrm{~K}^{-1} \mathrm{~km}^{-1} \mathrm{~s}$ (purple dashed line, Fig. 4 

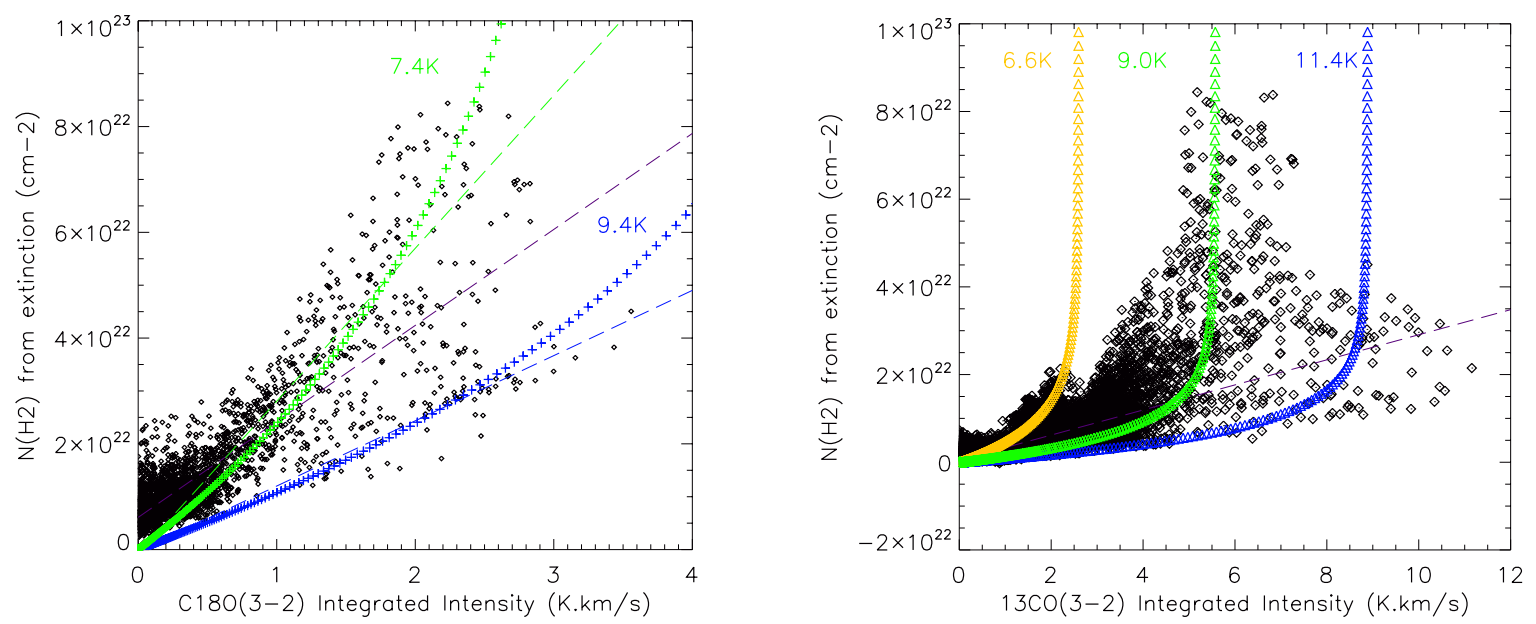

Fig. 4. Scatter plots of the $\mathrm{H}_{2}$ column density derived from the extinction map against the $\mathrm{C}^{18} \mathrm{O}($ left $)$ and ${ }^{13} \mathrm{CO}($ right $)$ integrated intensity $\left(\int T_{\mathrm{mb}}^{*} \mathrm{~d} v \mathrm{~K} \mathrm{~km} \mathrm{~s}^{-1}\right)$ for each pixel in the map. The purple dashed-lines represent the linear fit of the data points, with an upper limit constraint on the column density for the case of ${ }^{13} \mathrm{CO}$. Overlaid on the scatter plots are the predictions of RADEX models at different gas temperatures (on the left: $7.4 \mathrm{~K}$ in green and $9.4 \mathrm{~K}$ in blue crosses; on the right: $6.6 \mathrm{~K}$ in yellow, $9.0 \mathrm{~K}$ in green and $11.4 \mathrm{~K}$ in blue triangles). For the $\mathrm{C}^{18} \mathrm{O}$ (left) the green and blue dashed lines represent the linear approximations to the respective colour-coded RADEX model.

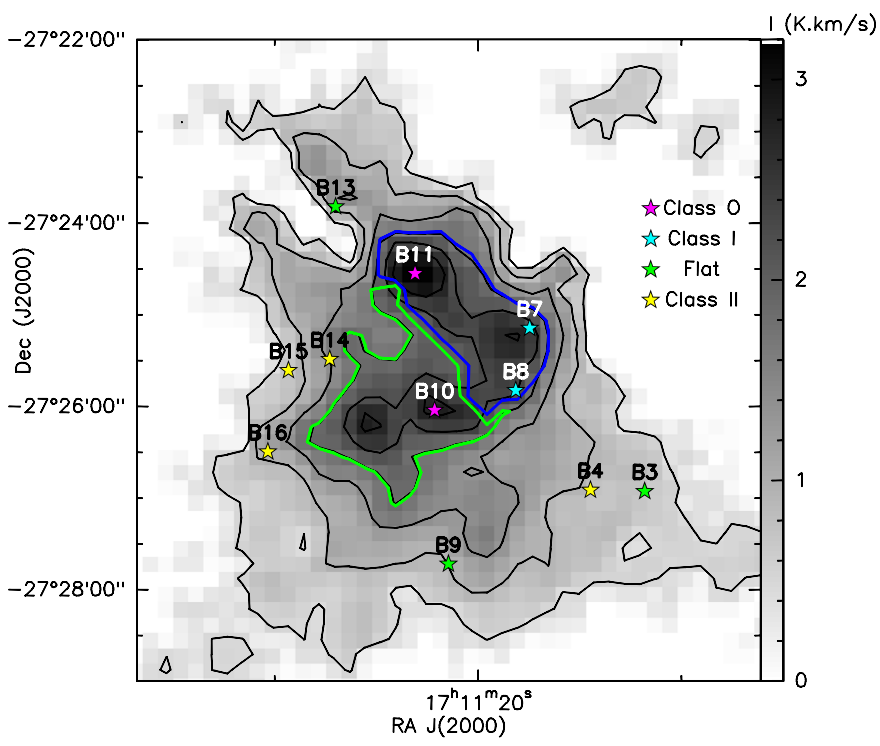

Fig. 5. Integrated intensity of $\mathrm{C}^{18} \mathrm{O}(3-2)$ in the central region of $\mathrm{B} 59$ (gray scale and contours). The protostars are marked with star symbols, and labeled following Brooke et al. (2007). The green and blue contoured regions correspond to the regions identified from the $\mathrm{C}^{18} \mathrm{O}$ scatter plot (see text and Fig. 4 left panel), and the green and blue departures of the ${ }^{13} \mathrm{CO}$ integrated emission. Though adjacent, these two regions have a temperature difference of $\sim 2 \mathrm{~K}$, the blue region being warmer. Note that while the colder green region hosts a single protostar (B10) and a "starless" $\mathrm{C}^{18} \mathrm{O}$ peak, the warmer blue region has three embedded protostellar objects (B11, B7 and B8).

left panel), which will be used to estimate the masses for the bulk of the cloud. However, points above and below the purple line, with $I_{\mathrm{C}^{18} \mathrm{O}}>1.5 \mathrm{~K} \mathrm{~km} \mathrm{~s}^{-1}$, fall into two spatially distinct regions of B59 (Fig. 5, green and blue contoured regions respectively). These two regions are of particular interest due to their optical depth and temperature structure. To better estimate the masses and virial parameters in these two specific regions, we used a linear fit to the green and blue models, for $N\left(\mathrm{H}_{2}\right)<8 \times 10^{22} \mathrm{~cm}^{-2}$ and $I_{\mathrm{C}^{18} \mathrm{O}}<3 \mathrm{~K} \mathrm{~km} \mathrm{~s}^{-1}$, shown as blue and green dashed lines in Fig. 4 left panel. These correspond to $X_{\mathrm{C}^{18} \mathrm{O}}$ (green) $=2.89 \times 10^{22} \mathrm{~cm}^{-2} \mathrm{~K}^{-1} \mathrm{~km}^{-1} \mathrm{~s}$ and $X_{\mathrm{C}^{18} \mathrm{O}}$ (blue) $=$ $1.23 \times 10^{22} \mathrm{~cm}^{-2} \mathrm{~K}^{-1} \mathrm{~km}^{-1} \mathrm{~s}$.

For ${ }^{13} \mathrm{CO}$ we estimated $X_{{ }^{13} \mathrm{CO}}=2.85 \times 10^{21} \mathrm{~cm}^{-2} \mathrm{~K}^{-1} \mathrm{~km}^{-1} \mathrm{~s}$ by constraining the linear fit to pixels where $N\left(\mathrm{H}_{2}\right)<3 \times$ $10^{22} \mathrm{~cm}^{-2}$ (purple dashed line in Fig. 4 right panel). We use this value to estimate the mass of the bulk of the cloud, but note that it will only provide a lower limit in those regions that show significant departures from a linear relationship due to optical thickening.

\subsection{Optical depth of $\mathrm{C}^{18} \mathrm{O}$ and ${ }^{13} \mathrm{CO}$}

To measure the optical depth of the ${ }^{13} \mathrm{CO}$ and $\mathrm{C}^{18} \mathrm{O}$ and study its variation across the cloud, we have followed the method in Ladd et al. (1998). This uses the ratio of the integrated intensities, and assumes the same excitation temperature for both species. The method is quite insensitive to the line width assumed, and therefore we have adopted a velocity width of $1 \mathrm{~km} \mathrm{~s}^{-1}$ throughout.

The map of the ratio of ${ }^{13} \mathrm{CO}$ to $\mathrm{C}^{18} \mathrm{O}$ integrated intensity (not shown) has values ranging from 2 to 4 in the denser regions and approaching 9 towards the edges of the cloud. A linear fit to the pixel-by-pixel comparison of the two species suggest an abundance ratio of ${ }^{13} \mathrm{CO}$ to $\mathrm{C}^{18} \mathrm{O}$ of around 6.4. However this value is significantly affected by the denser regions where the ${ }^{13} \mathrm{CO}$ line becomes optically thick. Therefore, we adopt an abundance ratio $f$ of ${ }^{13} \mathrm{CO}$ with respect to $\mathrm{C}^{18} \mathrm{O}$, of $f=8.4$ (Frerking et al. 1982), which is more consistent with the ratio we find at the edge of the cloud. This ratio implies $\mathrm{C}^{18} \mathrm{O}$ optical depths ranging between 0.1 to 0.5 over most of the cloud. However an optical depth ranging between 0.5 to 1.25 is found towards the central clump (Fig. 6), consistent with the expected increase of opacity from the $\mathrm{C}^{18} \mathrm{O}$ scatter plot. Therefore, we will consider $\mathrm{C}^{18} \mathrm{O}$ to be optically thin throughout most of the cloud, but only marginally so towards the central cores.

\subsection{Excitation temperature structure}

Since the ${ }^{13} \mathrm{CO}$ peak emission is optically thick in the bulk of the cloud $\left(\tau_{13}>1\right)$, we estimate the excitation temperature 

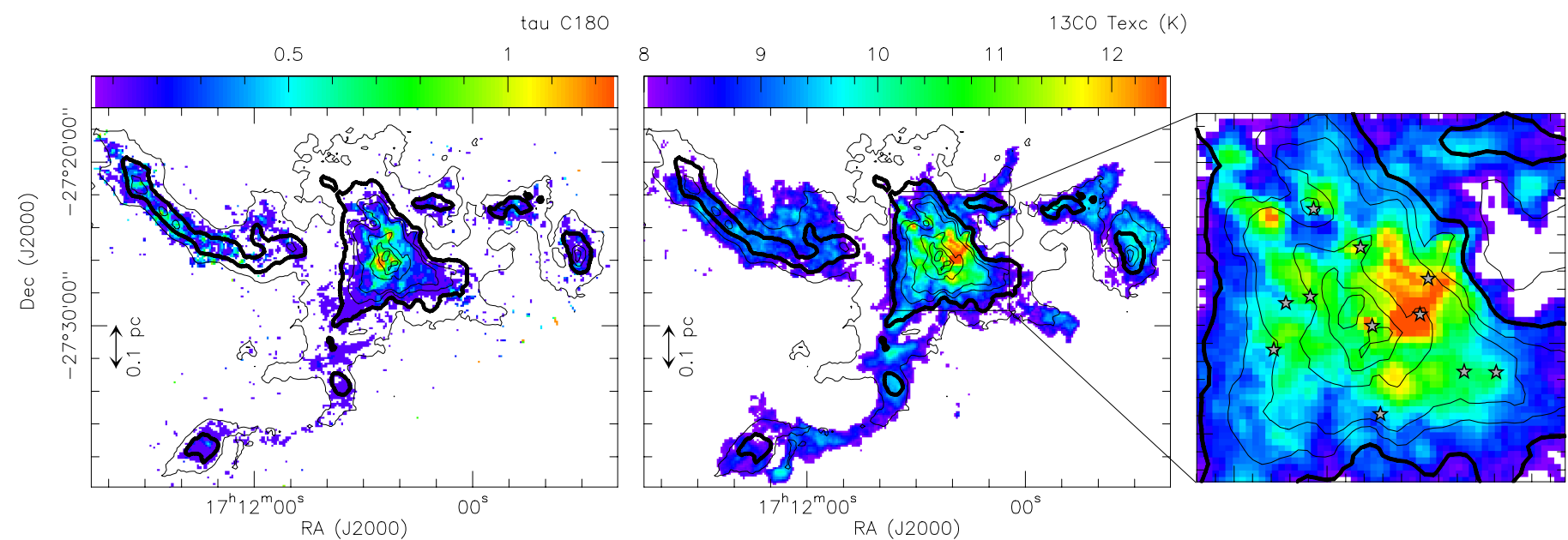

Fig. 6. Left: colour scale map of the $\mathrm{C}^{18} \mathrm{O}$ opacity calculated from the ratio of ${ }^{13} \mathrm{CO}$ to $\mathrm{C}^{18} \mathrm{O}$ emission. The $\mathrm{C}^{18} \mathrm{O}$ optical depth is less than 0.5 in most of the cloud and only in the central B59 core it rises to higher values, with three local peaks where it reaches values around unity. Centre and right: ${ }^{13} \mathrm{CO}$ excitation temperature map in colour scale. This shows an increase of temperature towards the central B59 (zoomed in on the right panel). In all panels, the contours are the dust extinction as in Fig. 1. The darker contour at $A_{v}=10$ which delineates the regions most likely to be in LTE.

of ${ }^{13} \mathrm{CO}$ assuming LTE conditions and optically thick emission. Using the radiative transfer equations under this assumption (e.g. Rybicki \& Lightman 1986), we have the relation:

$T_{\text {ex }}\left({ }^{13} \mathrm{CO}_{3-2}\right)=\frac{15.87}{\ln \left[1+15.87 /\left(T_{\max }\left({ }^{13} \mathrm{CO}\right)+0.045\right)\right]}$

where $15.87=h v / k$ for the frequency of ${ }^{13} \mathrm{CO} J=3 \rightarrow 2$, where $h$ is the Planck constant, $v$ is the frequency and $k$ is the Boltzmann constant. Here, $T_{\max }$ is the peak main beam temperature of the line. Where ${ }^{13} \mathrm{CO}$ is self absorbed, the peak used corresponds to the absolute maximum of the emission.

The resulting excitation temperature map is shown in Fig. 6, where we can see that most of the cloud is at $\sim 9 \mathrm{~K}$, and the star forming core is at $10-12 \mathrm{~K}$. Given that the ${ }^{13} \mathrm{CO}$ traces some of the outflow emission, in regions with self absorption we will be measuring the excitation temperature of the warmer outflowing gas. As such, outflow shocks can be seen in this image as local temperature maxima, even outside the B59 central region (for instance in the SE U-shaped ridge). Outside the contoured region shown in Fig. 6 the temperatures decrease to as low as $5 \mathrm{~K}$. However, in these diffuse ${ }^{13} \mathrm{CO}$ emission regions, not only is it likely that the ${ }^{13} \mathrm{CO}$ has lower optical depth, but also the densities are likely lower than the critical densities to maintain LTE, so the detailed results should be treated with caution.

However we can compare this temperature structure with what is predicted from the ${ }^{13} \mathrm{CO}$ scatter plots (right panel of Fig. 4). Firstly, we can identify three departures from the linear relation, explained by different gas temperatures (RADEX models in yellow, green and blue triangles). The first departure, at ${ }^{I^{13} \mathrm{CO}}=2 \mathrm{~K} \mathrm{~km} \mathrm{~s}^{-1}$, corresponds to the NE ridge of B59, and is consistent with very low temperatures (yellow triangles). The RADEX predictions indicate temperatures below $7 \mathrm{~K}$, while the LTE estimate (Fig. 6) indicates temperatures closer to $9 \mathrm{~K}$.

The other two departures, one where $I_{13} \mathrm{CO} \sim 6 \mathrm{~K} \mathrm{~km} \mathrm{~s}^{-1}$ and the other where $I_{13} \mathrm{CO}$ has its highest values, at column densities below $4 \times 10^{22} \mathrm{~cm}^{-2}$, correspond to the same two regions as those selected from the $\mathrm{C}^{18} \mathrm{O}$ scatter plot (contoured in Fig. 5). These are two adjacent portions in the central B59 region, each containing one of the two younger sources in the field (B10 and B11). We find that these two regions show a temperature difference of $\sim 2 \mathrm{~K}$ in both molecules (green and blue models in Fig. 4). Note that the warmer (blue) region also includes another two more evolved protostars (B7 and B8). This temperature difference inside the central region is consistent with our LTE ${ }^{13} \mathrm{CO}$ excitation temperature estimate shown in Fig. 6. The increase of temperature in the central region is associated with the position of the protostars, and it is likely due to the combined heating effect from radiative and outflow feedback from these young sources. However, the importance of radiative feedback in influencing the temperature is only local (within less than $10000 \mathrm{AU}$ from the protostars), and even though this could change the fragmentation properties of the gas at these scales, it likely does not substantially alter the fragmentation at the scale of the B59 star forming clump (i.e. $\sim 0.3 \mathrm{pc}$ ).

\section{Outflows}

\subsection{Identification of individual outflows}

To identify the population of protostellar outflows in B59, and estimate both their global and individual physical properties and energetics, we made use of our ${ }^{12} \mathrm{CO}$ mapping observations. These reveal a number of outflows bursting from the central sources of B59. The lefthand panels of Fig. 7 show the ${ }^{12} \mathrm{CO}$ blue and red emission from the larger scale outflows in B59. Here, we see relatively compact outflow emission bursting to the NE of the central region with spatially coincident red and blue emission (best seen on the righthand panels of Fig. 7). This emission falls in the extinction Cavity B (Fig. 1) and the outflow appears to trace back to one of the youngest sources in the field, the Class 0/I source B11.

Another set of larger scale outflow features, including a series of blue knots, and a red arc can be seen to the SE U-shaped ridge. The zoom-in of the central region (right panels) show that the driving source of the outflow responsible for the southern blue knot seems to be the other young Class 0/I source in the field, source B10. On the other hand, the red arc seems to be part of the outflow bursting from an older source (source B9).

This panel also shows that the two Class I sources (B7 and $\mathrm{B} 8$, light blue stars) sit on a region where the ${ }^{12} \mathrm{CO}$ from 

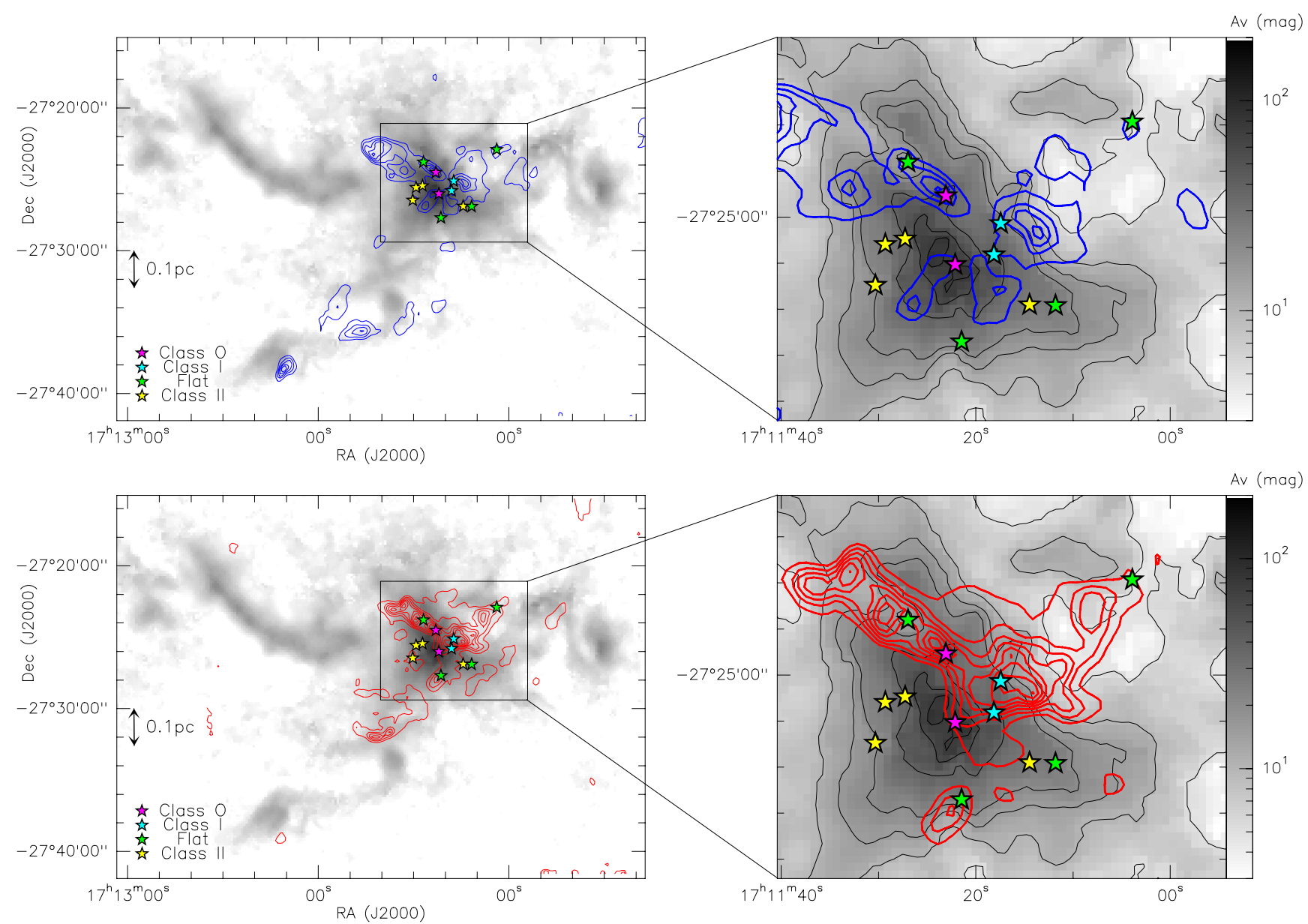

Fig. 7. ${ }^{12} \mathrm{CO}$ outflow emission in B59 shown as blue and red contours, together with the YSOs positions plotted on the extinction map from Fig. 1 in gray scale (and black contours on the righthand panels). The lefthand panels show the high velocity emission over the entire B59 region, while the right panels show a zoom into the central star-forming core. The ${ }^{12} \mathrm{CO}$ emission was integrated from -5 to $2.7 \mathrm{~km} \mathrm{~s}{ }^{-1}$ (blue) and from 4.2 to $15 \mathrm{~km} \mathrm{~s}^{-1}$ (red). The contours are from $3 \mathrm{~K} \mathrm{~km} \mathrm{~s}^{-1}$ on the left panels and from $4 \mathrm{~K} \mathrm{~km} \mathrm{~s}^{-1}$ on the right panels, in steps of $2 \mathrm{Kkm} \mathrm{s}^{-1}$.

the outflows of the two younger sources (purple stars) start to become confused. Therefore, despite the fact that some of the ${ }^{12} \mathrm{CO}$ wing emission in this particular region is likely to be driven by these two Class I sources, it is hard to disentangle from the outflows of the other nearby protostars. Finally, the flat spectrum sources B1/B2 (the western-most green star) also seem to show some signs of outflow emission. Though not very high velocity nor very strong emission, these outflow lobes (in particular the red lobe) fall near dust Cavity C.

For many of these outflow features, we cannot clearly identify a counter flow lobe. This is likely due to confusion of the more compact outflows in the central region. For this reason, in this paper we will focus on the properties of the unconfused outflows as well as on the global outflow properties. Interferometry will be important for disentangling these compact outflows, identifying their driving sources, and provide a better estimate of their energetics.

\subsection{Outflow inclination angles}

Before estimating the momentum, momentum flux and energy of the outflows, the observed velocities need to be corrected for the outflow inclination angle $i$ (where $i=0^{\circ}$ is defined to be along the line of sight). Observationally, it is hard to infer the inclination angle of the outflows with precision, and this introduces a critical source of uncertainty, as the velocities (and momentum) will be affected by a factor of $1 / \cos (i)$, the energies, by a factor $1 / \cos ^{2}(i)$, and the momentum flux, by a factor $\sin (i) / \cos ^{2}(i)$.

Nevertheless, for a few particular outflows in B59, a careful comparison of the blue-shifted and red-shifted emission can shed some light on the overall inclination of the flows. In particular, Flow 1 (Fig. 8) powered by B11 shows overlapping blue and red emission, consistent with being a single outflow lobe, close to the plane of the sky. Because the observed maximum velocities along the line of sight for the blue and red wings are similar, this constrains the outflow axis to being in the plane of the sky with less than $5^{\circ}$ uncertainty (otherwise the blue and red absolute velocities would differ by more than a factor 2 ). In addition, the projected opening angle of this outflow is $\sim 30^{\circ}$, implying that each of the outflow cone walls are inclined by $\sim 15^{\circ}$ with respect to the plane of the sky, giving $i \sim 75^{\circ}$. Note that if the outflow axis changes by $5^{\circ}$, the inclination angles would change, but while one would increase to $80^{\circ}$, the other would decrease to $70^{\circ}$. Because the mass, extent and velocities of the two lobes are similar, doing so would increase the estimate of the momentum and energy by a factor of 3 . Therefore, $i \sim 75^{\circ}$ for Flow 1 , remains a rather conservative value.

For Flow 3, the large spatial extent of the collimated blue knot (with a faint red counterpart at the end), combined with 
Table 1. Outflow properties.

\begin{tabular}{lccccc}
\hline \hline & $\begin{array}{c}M \\
\left(M_{\odot}\right)\end{array}$ & $\begin{array}{c}p_{\text {out }} \\
\left(M_{\odot} \mathrm{km} \mathrm{s}^{-1}\right)\end{array}$ & $\begin{array}{c}p_{\text {out }}^{\text {corr }} \\
\left(M_{\odot} \mathrm{km} \mathrm{s}^{-1}\right)\end{array}$ & $\begin{array}{c}E_{\text {out }} \\
\left(M_{\odot} \mathrm{km}^{2} \mathrm{~s}^{-2}\right)\end{array}$ & $\begin{array}{c}E_{\text {out }}^{\text {corr }} \\
\left(M_{\odot} \mathrm{km}^{2} \mathrm{~s}^{-2}\right)\end{array}$ \\
\hline Blue & 0.59 & 0.83 & 3.21 & 0.81 & 12.1 \\
Red & 0.63 & 0.55 & 2.13 & 0.40 & 5.97 \\
\hline
\end{tabular}

Table 2. Momentum flux of individual outflows.

\begin{tabular}{lccccccccccc}
\hline \hline & $\begin{array}{c}\text { Driving } \\
\text { source }\end{array}$ & $\begin{array}{c}l \\
(\mathrm{pc})\end{array}$ & $\begin{array}{c}v_{\max } \\
\left(\mathrm{km} \mathrm{s}^{-1}\right)\end{array}$ & $\begin{array}{c}i \\
\left({ }^{\circ}\right)\end{array}$ & $\begin{array}{c}l^{\text {corr }} \\
(\mathrm{pc})\end{array}$ & $\begin{array}{c}v_{\max }^{\text {corr }} \\
\left(\mathrm{km} \mathrm{s}^{-1}\right)\end{array}$ & $\begin{array}{c}t_{\mathrm{d}} \\
\left(\times 10^{3} \mathrm{yr}\right)\end{array}$ & $\begin{array}{c}M \\
\left(M_{\odot}\right)\end{array}$ & $\begin{array}{c}p_{\text {out }}^{\text {corr }} \\
\left(M_{\odot} \mathrm{km} \mathrm{s}^{-1}\right)\end{array}$ & $\begin{array}{c}E_{\text {out }}^{\text {corr }} \\
\left(M_{\odot} \mathrm{km}^{2} \mathrm{~s}^{-2}\right)\end{array}$ & $\begin{array}{c}F_{\text {out }}^{\text {corr }} \\
\left(M_{\odot} \mathrm{km} \mathrm{s}^{-1} \mathrm{yr}^{-1}\right)\end{array}$ \\
\hline Flow 1 - Blue & B11 & 0.19 & -5.5 & 75 & 0.20 & -21.3 & 9.2 & 0.15 & 0.97 & 4.36 & $1.1 \times 10^{-4}$ \\
Flow 1 - Red & B11 & 0.11 & 5.0 & 75 & 0.11 & 19.3 & 5.6 & 0.14 & 0.65 & 2.89 & $1.2 \times 10^{-4}$ \\
Flow 2 - Red & B9 & 0.23 & 5.5 & $57^{*}$ & 0.27 & 10.1 & 26.1 & 0.22 & 0.36 & 0.46 & $1.3 \times 10^{-5}$ \\
Flow 3 - Blue & B10 & 0.45 & -4.0 & 75 & 0.47 & 15.5 & 29.6 & 0.17 & 0.88 & 3.11 & $2.9 \times 10^{-5}$ \\
\hline
\end{tabular}

Notes. ${ }^{(*)}$ Flow 2 does not show a spatial overlay of the blue and red wings, indicative of it not being as close to the plane of the sky as flows 1 and 3. We therefore adopt a correction factor of 2.9 for the momentum flux of Flow 2 (i.e. $i \approx 57^{\circ}$ ), based what would be expected from a random distribution of inclination angles (Cabrit \& Bertout 1992; Bontemps et al. 1996). If instead an inclination of $75^{\circ}$ was adopted, the momentum flux of Flow 2 would increase by a factor 5 .

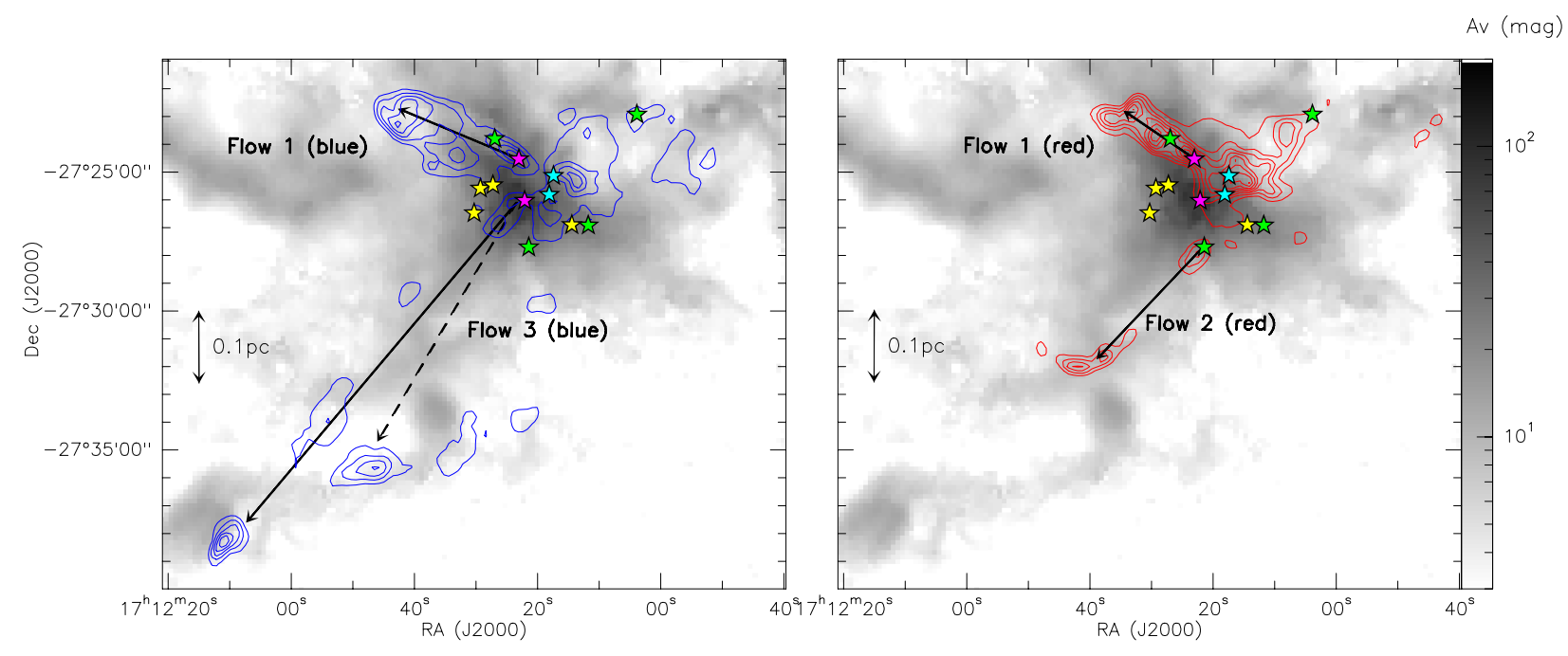

Fig. 8. Contours of the ${ }^{12} \mathrm{CO}$ blue-shifted and red-shifted emission (left and right panels respectively). Contours start at $3 \mathrm{~K} \mathrm{~km} \mathrm{~s}^{-1}$ for the blue emission and at $4 \mathrm{~K} \mathrm{~km} \mathrm{~s}^{-1}$ for the red, with steps of $2 \mathrm{~K} \mathrm{~km} \mathrm{~s}^{-1}$, overlaid on the extinction map of B59 as from Fig. 1 in gray scale. The black arrows show the different individual outflows used to calculate individual outflow properties: Flow 1 (blue and red), Flow 2 (red) and Flow 3 (blue).

a young driving source (B10) and a small velocity offset from the ambient cloud, also favours an outflow close to the plane of the sky. For simplicity, we will adopt the same inclination angle as for Flow 1. Considering the preferential alignment of the stronger outflows close to the plane of the sky, we also adopt $i \sim 75^{\circ}$ to estimate the global outflow properties.

\subsection{Outflow properties}

Estimating the mass of high velocity gas in the outflows, requires correcting for the optical depth of the ${ }^{12} \mathrm{CO}$ linewings, which can be done using the ${ }^{13} \mathrm{CO}$ data. Under LTE and assuming similar excitation temperatures, a fractional abundance of ${ }^{12} \mathrm{CO}$ relative to ${ }^{13} \mathrm{CO}$ of 62 (Langer \& Penzias 1993), and that the ${ }^{13} \mathrm{CO}$ wing emission is optically thin (Cabrit \& Bertout 1990), the optical depth of the ${ }^{12} \mathrm{CO}\left(\tau_{12}\right)$ can be determined from the ratio of the integrated wing emission of the two isotopologues.
We integrated the emission below $2.7 \mathrm{~km} \mathrm{~s}^{-1}$ for the blue wing and above $4.2 \mathrm{~km} \mathrm{~s}^{-1}$ for the red. For each wing, we then apply the correction factor of $\tau_{12} /\left(1-\mathrm{e}^{-\tau_{12}}\right)$ for the column density estimate at each pixel (Cabrit \& Bertout 1990; Curtis et al. $2010 \mathrm{~b}$ ). Assuming a kinetic temperature of the outflows of $25 \mathrm{~K}$ (twice the temperature of the dense gas), a CO abundance with respect to $\mathrm{H}_{2}$ of $10^{-4}$ and a distance of $130 \mathrm{pc}$, the gas mass can be written as

$M=1.78 \times 10^{-6} N_{\text {pix }}\left\langle\int T_{\text {mb }} \mathrm{d} v\right\rangle$

where $M$ is the gas mass in unit of $M_{\odot}$ assuming a molecular weight of $2.33, N_{\text {pix }}$ is the number of pixels in the outflows, and $\left\langle\int T_{\mathrm{mb}} \mathrm{d} v\right\rangle$ is the average integrated intensity, after the opacity correction of the $T_{\mathrm{mb}}$ at each pixel. If a higher temperature of $50 \mathrm{~K}$ was adopted for the outflows (as found by, e.g. Hatchell et al. 1999), our mass estimate would increase by $20 \%$. 

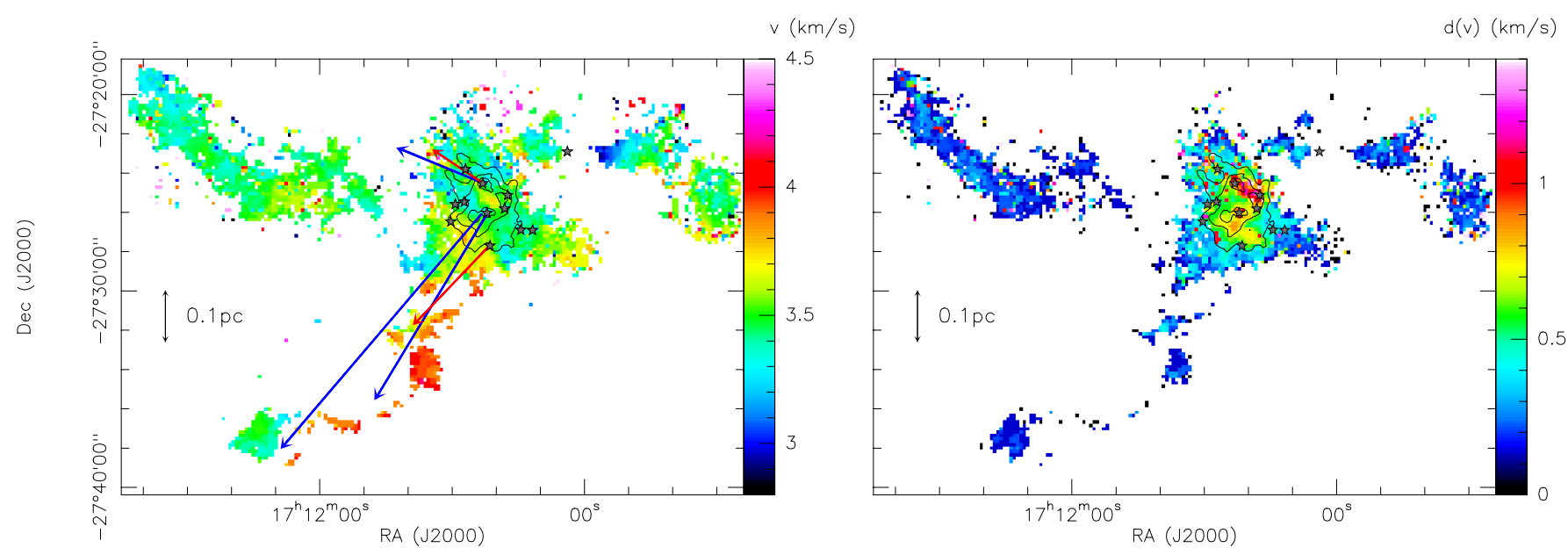

Fig. 9. $\mathrm{C}^{18} \mathrm{O}$ moment maps: mean velocity on the left panel, and velocity dispersion (FWHM) on the right, in colour scale overplotted with the integrated intensity in contours, and with the sources plotted as grey stars. The outflows identified in Fig. 8 are also indicated as blue and red arrows on the left-hand panel for comparison with the local velocity fields.

To estimate the average momentum, $p_{\text {out }}$, and kinetic energy, $E_{\text {out }}$, of the outflows, as well as the individual outflow momentum flux, $F_{\text {out }}$, we use

$$
\begin{aligned}
p_{\text {out }} & =\int m(v)\left|v-v_{0}\right| \mathrm{d} v \\
E_{\text {out }} & =\frac{1}{2} \int m(v)\left(v-v_{0}\right)^{2} \mathrm{~d} v . \\
F_{\text {out }} & =p_{\text {out }} / t_{\mathrm{d}}
\end{aligned}
$$

where $v_{0}$ is the velocity of the driving source, $m(v)$ the mass corrected for the optical depth, and $t_{\mathrm{d}}$ is the dynamical time of the outflow, defined as $t_{\mathrm{d}}=l / v_{\max }, l$ being the length of the flow, and $v_{\max }$ the maximum velocity of the flow.

The global momentum and energy of the outflows are summarised in Table 1, where $M$ is the gas mass contained in each of the outflow lobes, $p_{\text {out }}$ is the momentum and $E_{\text {out }}$ is the kinetic energy, shown here both without a correction for inclination angle, and assuming an inclination angle of $75^{\circ}\left(p_{\text {out }}^{\text {corr }}\right.$ and $\left.E_{\text {out }}^{\text {corr }}\right)$.

Table 2 shows the individual momentum flux for the four main flows of B59 (Fig. 8). In the table $l$ is the projected length of the outflow in the plane of the sky, $i$ is the assumed inclination angle, $v_{\max }$ is the line-of-sight maximum velocity with respect to the cloud's ambient velocity of $3.5 \mathrm{~km} \mathrm{~s}^{-1}$. The following columns give the remaining outflow properties, corrected for the inclination angle. The momentum flux of Flow 1 is higher than the momentum flux of both Flows 2 and 3 and is consistent with the expected values for Class 0 sources (i.e. $\sim 10^{-4} M_{\odot} \mathrm{km} \mathrm{s}^{-1} \mathrm{yr}^{-1}$, Bontemps et al. 1996; Curtis et al. $2010 \mathrm{~b})$. Flows 2 and 3 have momentum fluxes similar to those of a Class I protostar (i.e. $\sim 10^{-5} M_{\odot} \mathrm{km} \mathrm{s}^{-1} \mathrm{yr}^{-1}$, Bontemps et al. 1996; Curtis et al. 2010b).

\section{Ambient cloud}

\subsection{Dynamics}

To study the dynamics of the ambient gas of B59, we use the combined information from all three CO isotopologues. With the $\mathrm{C}^{18} \mathrm{O}$ emission in particular, we investigate the velocity gradients and changes in the velocity dispersion across the region (Fig. 9). The velocity map of the region (left panel of Fig. 9) shows that the NE ridge is uniform in velocity, at around $3.5 \mathrm{~km} \mathrm{~s}^{-1}$. Not only do we find little variation in velocity in this ridge, but also the linewidth is very small (with a FWHM as low as $0.3 \mathrm{~km} \mathrm{~s}^{-1}$, right panel of Fig. 9).

The U-shaped ridge (Fig. 1) shows a more complex velocity structure, with higher velocities than the rest of the cloud (at around $4 \mathrm{~km} \mathrm{~s}^{-1}$ ). This is somewhat surprising, since this edge correlates with a blue outflow knot from Flow 3 (Fig. 7). This strengthens the idea that this is an outflow close to the plane of the sky where the redshifted U-shape shows the compressed material behind the outflow, although there are no signs of the linewidth broadening in this ridge (right panel of Fig. 9).

The red arc from Flow 2 is also correlated with a $\mathrm{C}^{18} \mathrm{O}$ and extinction arc, delineating the righthand side of the U-shape. This $\mathrm{C}^{18} \mathrm{O}$ gas has higher velocities than the centre of B59, consistent with being pushed by the red-shifted Flow 2 which has a smaller inclination angle than Flows 1 and 3 .

In the central clump of B59 there is a velocity gradient of about $1 \mathrm{~km} \mathrm{~s}^{-1}$ across $0.1 \mathrm{pc}$ (left panel of Fig. 9). There is also a local increase of the linewidth, with the velocity dispersion (FWHM) ranging from $0.5 \mathrm{~km} \mathrm{~s}^{-1}$ to $1.2 \mathrm{~km} \mathrm{~s}^{-1}$.

This increase of linewidth can also be seen through positionvelocity (PV) diagrams (Figs. 10 and 11). The first cut (PV 1) is along the axis of Flows 2 and 3. On the left panels of Fig. 11 we can see the blue and red outflow knots from Flow 3 at $\sim 2.1^{\prime}$ offset as well as the red knot of Flow 2 at $\sim 11.5^{\prime}$ offset, in ${ }^{12} \mathrm{CO}$ and ${ }^{13} \mathrm{CO}$. The velocity structure of the U-shaped ridge is seen from offsets $3^{\prime}$ to $14^{\prime}$ where, while the eastern end is described by velocities $\sim 3.2 \mathrm{~km} \mathrm{~s}^{-1}$, the U-shaped and western end have stronger emission at $\sim 4 \mathrm{~km} \mathrm{~s}^{-1}$ (cf. Fig. 9). The ${ }^{13} \mathrm{CO}$ traces these two velocities throughout the ridge, but in fact, the U-shape itself is correlated with the red component (centre-left panel of Fig. 10). Cavity A is therefore also seen as a cavity in the lowvelocity redshifted ${ }^{13} \mathrm{CO}$ emission. The central region of B59 is delimited by a sharp edge (at an offset of $20.5^{\prime}$ ), where the emission from $\mathrm{C}^{18} \mathrm{O}$ is broad, and after which the emission drops sharply down to the noise in Cavity $\mathrm{C}$. This cavity, seen in extinction and with both ${ }^{13} \mathrm{CO}$ and $\mathrm{C}^{18} \mathrm{O}$, is delimited on both sides with high velocity outflowing gas.

The right column of Fig. 11 shows the cut through the NE ridge of B59 (PV 2, from 1' to $12^{\prime}$ offset), confirming the small linewidths and constant velocity throughout. The emission from the central portion of B59 is seen at offsets between 14' 
Dense Gas: $\mathrm{C} 180(3-2)$

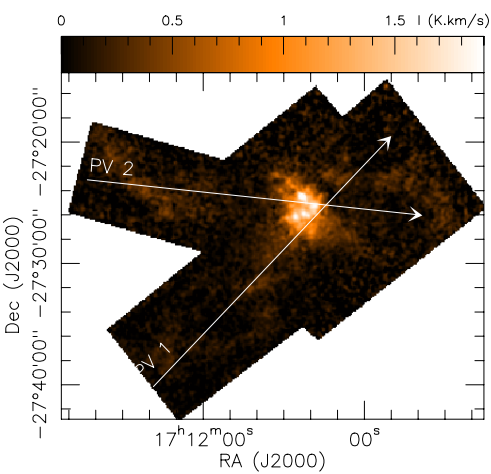

Intermediate densities: $13 \mathrm{CO}(3-2)$

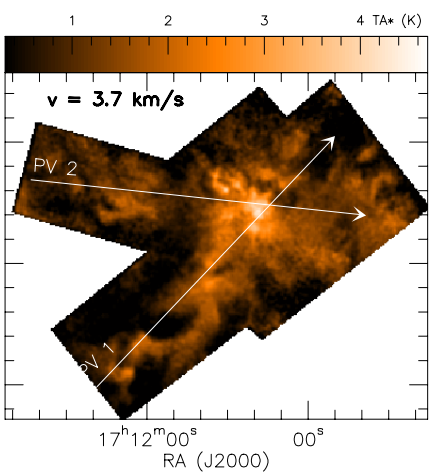

Outflow emission: $12 \mathrm{CO}(3-2)$

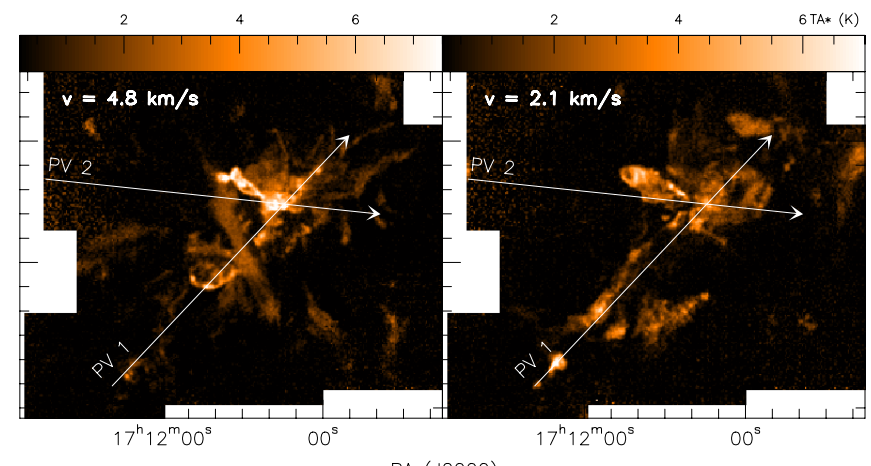

Fig. 10. Left: integrated intensity $\left(\int T_{\mathrm{A}}^{*} \mathrm{~d} v\right)$ map of $\mathrm{C}^{18} \mathrm{O}$ showing the distribution of the dense gas in B59; centre-left: ${ }^{13} \mathrm{CO}$ channel map at $v=3.7 \mathrm{~km} \mathrm{~s}^{-1}$ (slightly red-shifted with respect to the cloud's ambient velocities of $3.5 \mathrm{~km} \mathrm{~s}^{-1}$ ), tracing the intermediate density gas, and showing some of the cavities discussed in the text; centre-right and right: ${ }^{12} \mathrm{CO}$ channel maps at $v=4.8 \mathrm{~km} \mathrm{~s}^{-1}$ and $v=2.1 \mathrm{~km} \mathrm{~s}{ }^{-1}$ respectively, illustrating the red-shifted and blue-shifted outflows of the region. The position of the two position-velocity $(\mathrm{P}-\mathrm{V})$ diagrams of Fig. 11 are shown as white arrows and labeled as PV 1 and PV 2 in the four panels.
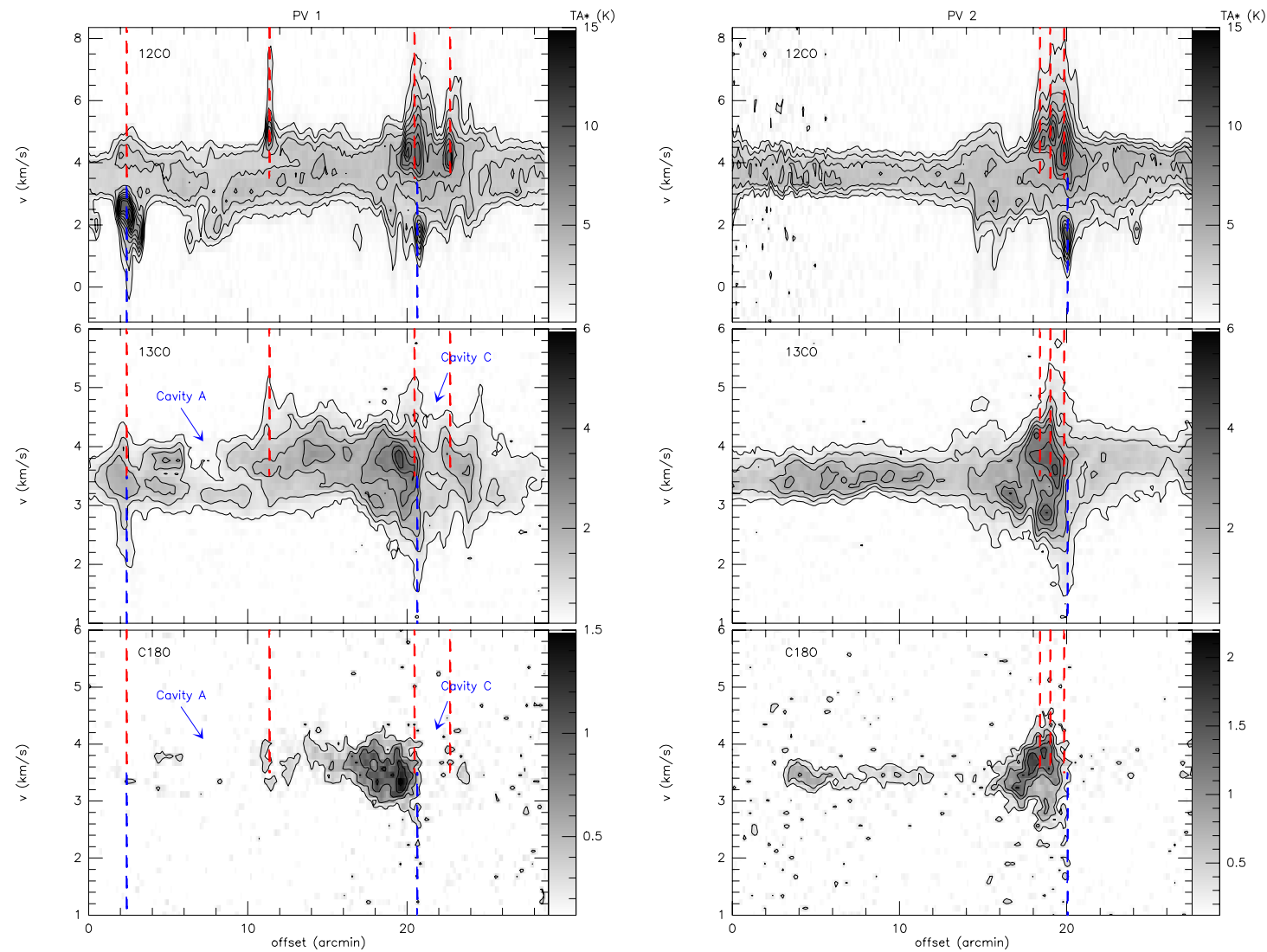

Fig. 11. P-V diagrams of $\mathrm{B} 59$ in ${ }^{12} \mathrm{CO}$ (top panels), ${ }^{13} \mathrm{CO}$ (centre) and $\mathrm{C}^{18} \mathrm{O}$ (lower panels). Each column is the $\mathrm{P}-\mathrm{V}$ cut as labeled at the top and as shown in Fig. 10. The position of some of the outflows are indicated with blue and red dashed lines and cavities are also indicated. The contours are from $1 \mathrm{~K}$ with steps of $1 \mathrm{~K}$ for ${ }^{12} \mathrm{CO}$, from $0.3 \mathrm{~K}$ with steps of $0.6 \mathrm{~K}$ for ${ }^{13} \mathrm{CO}$ and from $0.25 \mathrm{~K}$ with steps of $0.3 \mathrm{~K}$ for $\mathrm{C}^{18} \mathrm{O}$ (in $T_{\mathrm{A}}^{*}$ ).

and $20^{\prime}$ and, similarly to PV 1 , is delimited by a sharp edge to the west (leading to the same Cavity $\mathrm{C}$ as seen in PV 1). The $\mathrm{C}^{18} \mathrm{O}$ emission shows significant local broadening and the velocities agree with those of ${ }^{13} \mathrm{CO}$. In particular, the red outflow wings correlate with regions where the $\mathrm{C}^{18} \mathrm{O}$ is broader with stronger red emission. The marked blue outflow wing can be tentatively seen with $\mathrm{C}^{18} \mathrm{O}$ but it approaches the noise level of the data. Nevertheless, the lower velocity blue shifted ${ }^{13} \mathrm{CO}$ emission at an offset of $\sim 20^{\prime}$ is also seen in the $\mathrm{C}^{18} \mathrm{O}$ PV diagram.

\subsection{The hierarchical structure}

To study the hierarchical structure of B59, we have used a dendrogram extraction technique developed by Rosolowsky et al. (2008). This method is useful to understand the different structures that lie within B59. However, like any other clumpextraction technique, it cannot be blindly used in a region. In the case of B59, most of the leaves retrieved are not "real" cores in the sense of structures which are progenitors of future or 
currently forming protostars. In fact, only two leaves in the central B59 correspond to actual star-forming cores. The remaining structures trace the two halves of outflow emission, or less dense material which may or may not be bound and form stars in the future. A detailed description of the extraction analysis and results which confirm the broken structure of B59 can be found in Appendix A. In summary:

- the SE U-shaped ridge is not a single structure but two different arch-like structures, consistent with two compressed fronts from two different outflows (Flows 2 and 3). This suggests that the gas once in Cavity A has been cleared by Flow 3 and compressed, forming the eastern side of the $\mathrm{U}$-shaped ridge;

- the Western cores are physically separated from the main B59 structure, consistent with the existence of a gas-deprived cavity (Cavity C). The presence of outflowing gas possibly associated with the B1/B2 system, which is seen at the edges of this cavity, suggests that this cavity was possibly cleared by the outflow itself earlier in the evolution of these sources;

- the NE ridge is, at present, a coherent structure, gravitationally bound, uniform in velocity, and with low velocity dispersion. It could be on the verge of forming future prestellar cores;

- and finally, the dendrogram analysis finds that the central region does not show evidence for further fragmentation into new cores, apart from the two cores surrounding the younger protostars in the field. All the other structures found in this central region are components of the outflowing gas, particularly along the walls of Flow 1.

\section{Discussion: outflow Interactions}

\subsection{Outflows and the cloud}

Our hierarchical study of the region also provides a reliable mass estimates throughout the different scales of the cloud. These can be used to estimate the binding energy at different size scales, which can be compared to the kinetic energy carried out by the outflows, to estimate their respective impact. The total kinetic energy of the outflows (Sect. 5) is $\sim 18 M_{\odot} \mathrm{km}^{2} \mathrm{~s}^{-2}$, assuming an inclination angle of $75^{\circ}$. We estimate the binding energy (i.e. the potential energy) of the dense material using the $\mathrm{C}^{18} \mathrm{O}$ masses. For $30 M_{\odot}$ and a radius of $0.30 \mathrm{pc}$ (Appendix A), and assuming a density profile $\propto r^{-2}$ (similar to that found by Román-Zúñiga et al. 2012), the gravitational potential energy is $12 M_{\odot} \mathrm{km}^{2} \mathrm{~s}^{-2}$. The outflows therefore carry an energy comparable to the binding energy of the dense material. However, the kinetic energy of the outflows may be deposited outside the cores, and therefore may not be disrupting the dense cores themselves.

In fact above we have shown that the outflows are correlated with the shapes and cavities seen in $\mathrm{C}^{18} \mathrm{O}$ and extinction outside the central region, indicating that the outflows are able to push, carve and shape the material (e.g. Sect. 6). For instance the material along the SE U-shaped ridge is a fossil structure from Flows 2 and 3. Its U-shape, its velocity structure, and its local temperature maxima are a result of the impact of outflows on an originally less dense medium.

\subsection{Outflows and the dense gas}

Despite the clear impact on larger scales of the outflows in the B59 cloud, their importance on the dense gas is harder to disentangle. For instance, in the denser central region of B59, we see temperature peaks likely due to the combined effect of radiation from the individual protostars, and outflow shocks from the ensemble of four protostars (Fig. 6). We detect a broadening of the $\mathrm{C}^{18} \mathrm{O}$ emission towards the central clump, which could be in principle be associated with a combination of rotation, shear, infall and outflow motions. But an upper limit for the contribution of infall to the linewidth can be estimated by assuming that the entire B59 central clump is in free fall collapse. This would result on an infall velocity of $\sim 0.2-0.3 \mathrm{~km} \mathrm{~s}^{-1}$ (generating a velocity dispersion of that order or less), insufficient to explain the observed line widths.

To investigate more closely the possible effect of the outflows on the line width of dense gas, we have studied the emission in an area where there is initial evidence for the interaction of the outflow with the dense gas (in the form of a cavity) and where no significant velocity gradients are detected. This is the case along Flow 1, which is an unconfused and well collimated outflow located in the densest part of B59 (Fig. 7). For this test case, we compared the average profiles of the ${ }^{12} \mathrm{CO}$ integrated intensity as a measure of the outflow strength and location; the visual extinction as a measure of the quantity of material along the line of sight; and the $\mathrm{C}^{18} \mathrm{O}$ linewidth as a measure of the velocity dispersion. To do this, we took several slices along a direction perpendicular to the outflow main axis.

Since the outflow profile changes along its length, we estimated the average profiles within two separate regions. These are shown as boxes in Fig. 12 (left panels) overplotted on the central region of $\mathrm{B} 59$ in the ${ }^{12} \mathrm{CO}$ integrated intensity (top), the extinction map (middle) and the $\mathrm{C}^{18} \mathrm{O}$ linewidth (bottom). These boxes are colour-coded as the respective profiles, shown in the other panels of the same figure. To serve as reference, the dashed lines show the position of the peak of ${ }^{12} \mathrm{CO}$ integrated intensity.

Closest to the driving source, inside the blue boxed region, the outflow is collimated and constrained to a $\sim 1^{\prime}$-wide region (top row, central panel of Fig. 12). In the second region, delimited by the green box, the outflow is broader (top row, right panel). Here, the blue shifted emission starts forming an arched lobe opening towards the east, while the the redshifted outflow ends with a sharp edge (Figs. 7 and 8). This sharp tip is immediately followed by a linewidth peak, reminiscent of an impacted outflow knot (Fig. 12, first column, lower panel).

In both boxes, the outflow falls in a similar-sized cavity in the extinction (middle panels). This Cavity B (Fig. 1) has clearly been cleared up by this powerful outflow. Figure 12 (lower row panels) shows the impact of the outflow on the dense gas velocity dispersion. Even though there is less $\mathrm{C}^{18} \mathrm{O}$ gas in this cavity, there is still enough material to detect an increase of linewidth along the outflow axis. Immediately before and after the outflow axis, towards higher column densities, the linewidths are still relatively high (when compared to the outer parts of B59), but decrease steadily moving away from the outflowing material. This is the case for the regions in the green box between $1^{\prime}$ and $3^{\prime}$, where the $\mathrm{C}^{18} \mathrm{O}$ linewidth decreases until it reaches our detection limit. This suggests that the outflows are having a direct impact on both the shape of the region, and the linewidths of the denser material in B59.

An upper limit on the contribution from the outflowgenerated turbulence in the total velocity dispersion of the region, can be estimated by assuming that the initial turbulence of the gas in B59 (before any outflow feedback) was as low as that currently found outside the central B59 $\left(0.3 \mathrm{~km} \mathrm{~s}^{-1} F W H M\right.$ in the NE ridge, cf. Fig. 9, and Tables A.1 and A.2), and that 

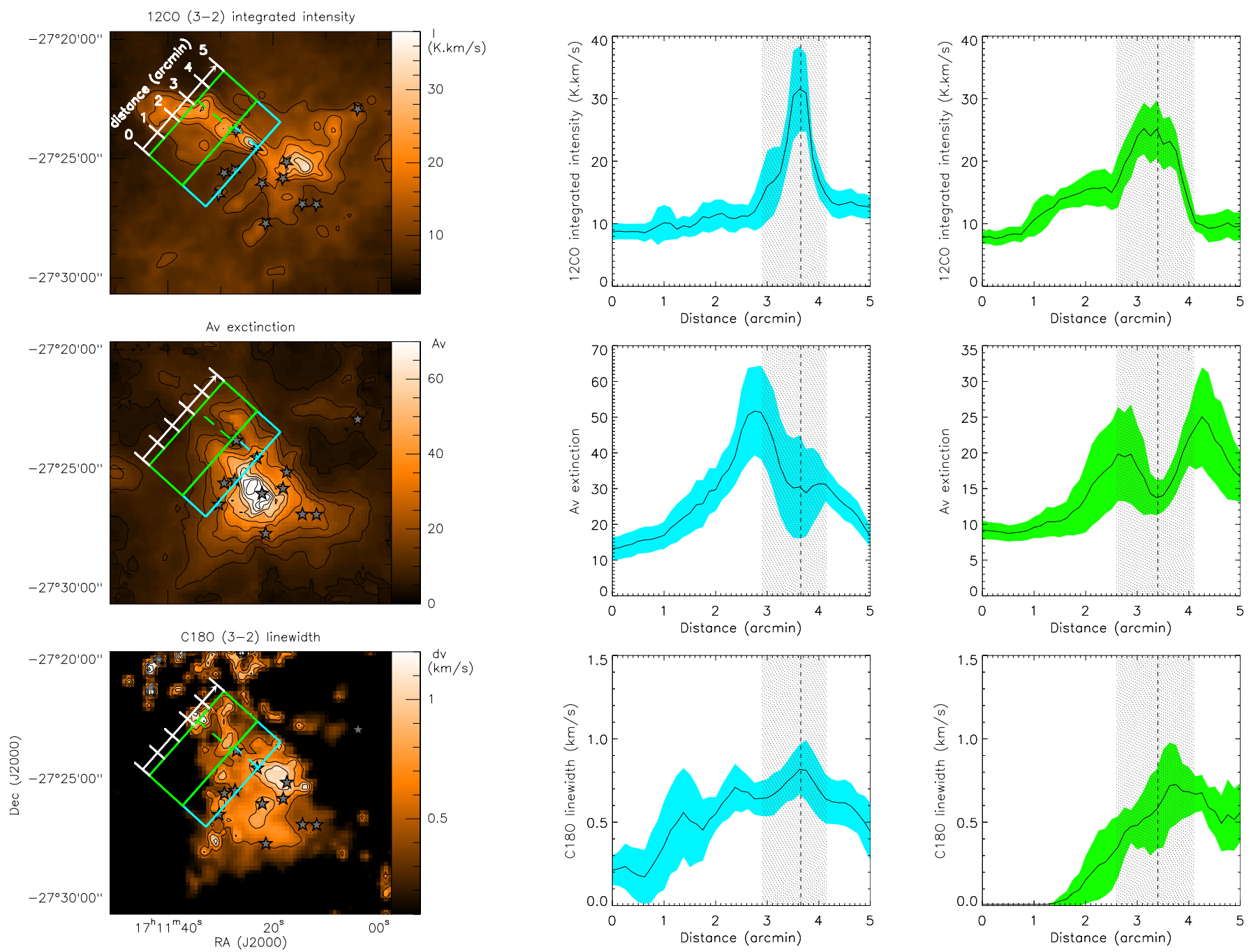

Fig. 12. Left column: close up of the central region of B59, where the colour scale and contours are the ${ }^{12} \mathrm{CO}$ integrated intensity map (top), the visual extinction map (centre) and the $\mathrm{C}^{18} \mathrm{O}$ linewidth map (bottom). The regions used to estimate the average profiles (shown in the centre and right columns) across Flow 1 are shown as blue and green boxes. The dashed lines show the position of the peak of ${ }^{12} \mathrm{CO}$ integrated intensity as averaged within each box. Centre and right columns: average profiles of ${ }^{12} \mathrm{CO}$ integrated intensity (top row panels), visual extinction (central row panels), and $\mathrm{C}^{18} \mathrm{O}$ linewidth (FWHM, bottom row panels). Each column corresponds to a different averaging box, colour-coded as in the left column. The dashed lines and gray-shadowed areas mark the peak and extent of the ${ }^{12} \mathrm{CO}$ profile as seen on the first row panels, respectively. The colour-coded shaded areas show the 1-sigma dispersion of each quantity.

the increase of linewidth along Flow 1 is solely due to outflowinjected turbulence. The maximum FWHM detected along Flow 1 is $\sim 1.0 \mathrm{~km} \mathrm{~s}^{-1}$, so we estimate that the outflow-generated turbulence in B59 is locally of the order of $0.9 \mathrm{~km} \mathrm{~s}^{-1} F W H M$ (i.e. $\sigma_{v}^{\text {out }} \sim 0.4 \mathrm{~km} \mathrm{~s}^{-1}$ ). This is, however, most certainly dependent on the power of the outflow and on the age and mass of the driving source.

Another way to assess the importance of the outflows as turbulence generators is to compare the turbulent kinetic energy and the outflow energy (similarly to e.g. Arce et al. 2010). In the central region, the kinetic energy is $E_{\text {turb }}=4 M_{\odot} \mathrm{km}^{2} \mathrm{~s}^{-2}$, while the outflow energy, estimated in the same area is $E_{\text {out }}=$ $0.62 M_{\odot} \mathrm{km}^{2} \mathrm{~s}^{-2}$, before applying any correction factor for outflow inclination. If correcting for a random distribution of angles and adopting $i \sim 57^{\circ}$, this becomes $E_{\text {out }}^{\text {corr }}=5.2 M_{\odot} \mathrm{km}^{2} \mathrm{~s}^{-2}$, or even $E_{\text {out }}^{\text {corr }}=9.2 M_{\odot} \mathrm{km}^{2} \mathrm{~s}^{-2}$ if assuming an inclination angle of $75^{\circ}$ as in Sect. 5. This suggests that the outflows carry more than enough energy to provide the turbulent support of the central B59. However, since the central region of B59 shows velocity gradients and is also likely affected by infall motions, it is not straightforward to conclude if the observed turbulence is predominantly generated by outflows.

Instead we focus on estimating the fraction of the outflow energy deposited in the immediate surroundings of Flow 1. Taking the regions in the outflow walls we estimate from the $\mathrm{C}^{18} \mathrm{O}$ that the total kinetic energy is $E_{\text {turb }}=0.9 M_{\odot} \mathrm{km}^{2} \mathrm{~s}^{-2}$. From the respective portion of Flow 1, we estimate that the outflow energy is $E_{\text {out }}^{\text {corr }}=2 M_{\odot} \mathrm{km}^{2} \mathrm{~s}^{-2}$. As mentioned in Sect. 5 a change in the inclination angle adopted for Flow 1 will increase the estimate of the outflow energy. This together with the fact that the total kinetic energy around the outflow does include other terms (such as the thermal and isotropic turbulence), suggests that only a fraction (less than half) of the energy carried by the outflow is deposited in the immediate surroundings consistent with, for example, Cunningham et al. (2006). The remaining energy is deposited outside the star forming core.

We stress that this is estimate is for the specific case of Flow 1 . This efficiency of outflow energy deposition will likely 
vary depending on the energy of the outflows and the density and velocity conditions of the regions with which the outflows interact.

\section{Summary and conclusions}

B59 is a star forming region with a small and young proto-cluster at its centre. In its vicinity the gravitationally bound NE ridge appears likely to form stars in the future. This ridge has a coherent velocity structure, but presently shows very little sub-structure. Another region which may eventually form stars are the western cores. However the mass reservoir available in this region is not very large and so if it does become active, it will likely form no more than a single low mass protostar/protostellar system.

All the protostars in $\mathrm{B} 59$ are located in a $\mathrm{C}^{18} \mathrm{O}$ twisted filament-like structure (not seen in the dust) in B59's centre. In this region, there is a sharp temperature increase of $2 \mathrm{~K}$ correlated with the position of the protostars, likely due to radiative heating and outflow feedback. Nevertheless, the heating is a very localised effect, not likely to substantially change the properties of the fragmentation of B59 as a whole.

We identify a number of cavities in the B59 region that are either correlated with outflowing gas, or adjacent to outflow knots. Finally, we investigated the relation between the outflowing gas and the linewidth of the denser gas and found a clear correlation with an increase of linewidth of $\mathrm{C}^{18} \mathrm{O}$ along the outflow axis.

Our study of B59 suggests that outflows are responsible for sweeping up and compressing gas as they make their way out of the cloud. We conclude that the outflows are interacting closely with the dense material broadening the linewidths of the material in the densest gas. Outside of the star-forming core, and as the outflows travel through a lower density material, their impact is seen as a re-shaping of the gas and dust that surrounds the protocluster. Their impact on the dense gas is shown to be an efficient source of turbulence that provides just enough support for the cloud against collapse at sub-parsec scales $(\sim 0.1-0.3 \mathrm{pc})$. The comparable kinetic and potential energies are consistent with the idea that the B59 core is long-lived and slowly collapsing. We, therefore, confirm that outflows are, as speculated by Covey et al. (2010) and Román-Zúñiga et al. (2012), potentially responsible for supporting the B59 clump against collapse.

A localised line broadening in the dense material is not commonly observed, since $\mathrm{C}^{18} \mathrm{O}$ does not trace high velocity outflowing gas. In B59, most of the gas originally had narrow line widths, and the turbulence input from outflows is more easily detected. In this context, B59 appears in marked contrast with regions such as Serpens (e.g. Duarte-Cabral et al. 2010), where no clear evidence for the outflows is seen in the $\mathrm{C}^{18} \mathrm{O}$ emission. Nevertheless, the actual linewidths of $\mathrm{C}^{18} \mathrm{O}$ in Serpens (homogeneously around $\sim 1-1.5 \mathrm{~km} \mathrm{~s}^{-1} F W H M$ ) are in fact similar to those in the central core of B59. Serpens being a much more dynamic region resulting from a collision of clouds (Duarte-Cabral et al. 2011) and with a large number of protostars powering outflows (more than 20 Class 0 and Class I sources in Serpens against four in B59), it may be that we are simply no longer able to detect the underlying quiescent gas in Serpens.

We suggest that outflows are an important source of turbulence in the gas around star forming cores, in agreement with the modeling work from e.g. Matzner (2007); Nakamura \& Li (2007). However, in regions where the initial dynamics of the gas implies intrinsically larger linewidths, the contribution from the outflow-generated turbulence can potentially become negligible. In fact, the final linewidth will only show a measurable broadening from the outflow contribution (of at least $30 \%$ ), when the injected turbulence is at least as large as the original initial turbulence of the gas. Assuming that the measured generated turbulence $\left(\sigma_{v}^{\text {out }} \sim 0.4 \mathrm{~km} \mathrm{~s}^{-1}\right)$ is close to the typical value of Class 0 protostellar outflows, the outflow broadening will only be detectable in regions where the original $\sigma_{v}$ of the gas is of the order of $0.4 \mathrm{~km} \mathrm{~s}^{-1}$ (or less). The relative support provided by such an input will also vary depending on the mass and the potential energy available to a given protocluster. In star-forming clumps embedded in more massive molecular clouds (i.e. lying in a deeper potential well), the gravitational energy will easily exceed such outflow-induced turbulent support, and the later will not be enough to halt a larger scale collapse.

We find that the energy carried by the outflows is larger than that needed to provide the current turbulent support of B59, and that only a fraction, less than half, of the energy of the outflows is efficiently converted into turbulent motions of the gas in the immediate surroundings of the outflows. The remaining energy is, therefore, deposited outside the star forming core. In regions denser than B59 this efficiency may increase, as the interaction of the outflowing gas and the environment will be more important. On the other hand, the balance of gravitational and turbulent energy will also be more important in such denser regions.

Acknowledgements. We thank the anonymous referee for his/her comments that helped making the paper clearer. We thank J. Pineda and E. Rosolowsky for support with using the dendrogram code. A.D.C. is supported by the project PROBeS funded by the French National Research Agency (ANR), and also acknowledges the support of a grant from the Fundação para a Ciência e a Tecnologia (FCT) de Portugal, under which part of this work was done. The data reduction and analysis was done using GILDAS software (http://www . iram. fr/IRAMFR/GILDAS) and Starlink software (http://starlink. jach. hawaii.edu/starlink). The James Clerk Maxwell Telescope is operated by the Joint Astronomy Centre (JAC) on behalf of the Science and Technology Facilities Council (STFC) of the United Kingdom, the Netherlands Organisation for Scientific Research and the National Research Council of Canada. This research used the facilities of the Canadian Astronomy Data Centre operated by the National Research Council of Canada with the support of the Canadian Space Agency.

\section{Appendix A: The hierarchical structure of B59}

\section{A.1. Method: dendrograms}

To study the cloud structure of B59, we used a dendrogram code developed and applied to star forming regions by Rosolowsky et al. (2008) that traces the hierarchical structure of the cloud, representing the cloud as a tree-like structure. This technique begins with the identification of all local maxima in a datacube (the leaves of the tree-like structure), and contours down the dataset, finding all the underlying structure that connects the different leaves together (i.e. the branches), down to the root. Each connecting point between two structures (leaves, branches and root) is identified as a node in the tree. The code we used to implement this dendrogram technique is fully described in Rosolowsky et al. (2008). This code also calculates several properties of the cloud at each contour level, such as the integrated intensity, the size of the structure, the velocity dispersion, the mean velocity and mean position.

We can calculate the masses within the dendrogram code by taking a linear relation between the $\mathrm{H}_{2}$ column density and the integrated intensity of the molecular line considered $\left(X_{\mathrm{CO}}\right)$, following the procedure described in Rosolowsky et al. (2008), where

$M=1.84 \times 10^{-20} X_{\mathrm{CO}} L_{\mathrm{CO}}$ 

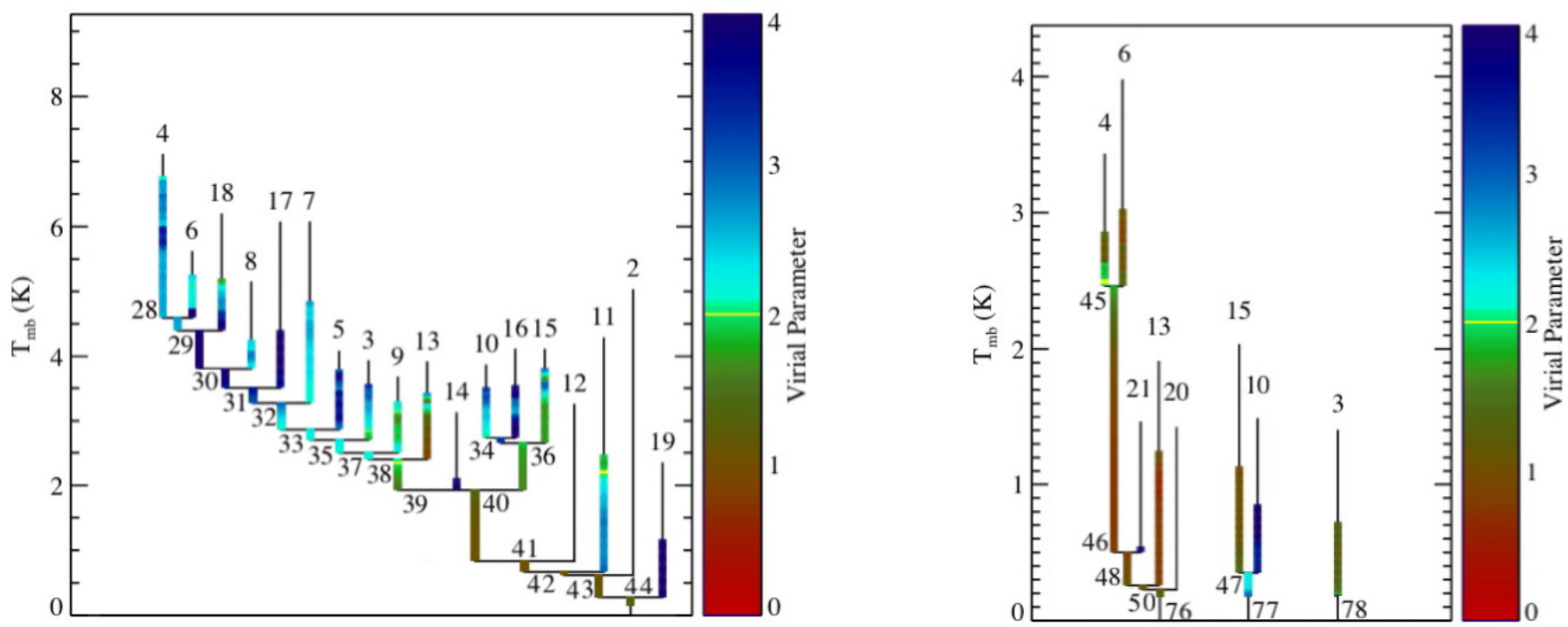

Fig. A.1. Dendrogram of the ${ }^{13} \mathrm{CO}($ left $)$ and $\mathrm{C}^{18} \mathrm{O}$ emission (right) in $\mathrm{B} 59$, with the virial parameter calculated for each contour. The regions where no virial parameter is shown (e.g., the tips of the leaves) are due to the lack of sufficient quality data to calculate the properties, such as insufficient number of pixels. The numbers refer to nodes and leaves as found by the code.

where $M$ is the $\mathrm{H}_{2}$ gas mass (in $M_{\odot}$ ) derived from the $\mathrm{CO}$ isotopologue in consideration, $X_{\mathrm{CO}}$ is the CO-to- $\mathrm{H}_{2}$ conversion factor described in Sect. 4.1 (in units of $\mathrm{cm}^{-2} \mathrm{~K}^{-1} \mathrm{~km}^{-1} \mathrm{~s}$ ), and $L_{\mathrm{CO}}$ is the luminosity from the considered $\mathrm{CO}$ emission (in $\mathrm{K} \mathrm{km} \mathrm{s}^{-1} \mathrm{pc}^{2}$ ). This luminosity $L_{\mathrm{CO}}$ is calculated as $L=F d^{2}$, where $d$ is the distance to the cloud and $F$ is the integrated flux of the region, i.e. the sum of all the emission in the region, calculated as $F=\sum_{i} T_{i} \delta \theta_{x} \delta \theta_{y} \delta v$. The value of $1.84 \times 10^{-20}$ arises from the units conversion, assuming a molecular weight of 2.33 .

The virial parameter $\alpha$ is estimated for each structure at each contour level from the virial balance between the kinetic and gravitational potential energy, i.e. $\alpha=2 E_{\mathrm{k}} / E_{\mathrm{g}}$. Assuming a uniform density profile, this translates into

$\alpha=\frac{5 \sigma_{v}^{2} R}{M G}$

where $\sigma_{v}$ is the velocity dispersion and $R$ is the size of the structure as estimated by the dendrogram code. Assuming a density profile of $\rho \propto r^{-2}$ would decrease the viral parameter by a factor of $3 / 5$. Without the presence of any additional terms in the virial equation (such as magnetic field or external pressure), virial equilibrium corresponds to $\alpha=1$. However, given the uncertainties associated with these assumptions, we consider a structure to be gravitationally bound when the virial parameter is less than 2. The use of such a parameter is best seen as an internal means of comparison of structures within the cloud rather than an absolute measure of the individual equilibrium states.

We performed this dendrogram analysis of B59 on the $3 \mathrm{D}$ datacubes of the intermediate density tracers ${ }^{13} \mathrm{CO}$ and $\mathrm{C}^{18} \mathrm{O}$. We considered the minimum height of a leaf to be one-sigma of the rms noise level, and chose the bijection method to calculate the cloud properties (similarly to Rosolowsky et al. 2008).

\section{A.2. Results}

\section{A.2.1. The ${ }^{13} \mathrm{CO}$ and $\mathrm{C}^{18} \mathrm{O}$ dendrograms}

The dendrograms for ${ }^{13} \mathrm{CO}$ and $\mathrm{C}^{18} \mathrm{O}$ are shown in Fig. A.1, left and right panels respectively. This hierarchical stratification of ${ }^{13} \mathrm{CO}$ finds the entire structure of $\mathrm{B} 59$ to be interconnected and part of the same cloud, while with $\mathrm{C}^{18} \mathrm{O}$ the cloud is no longer interconnected (likely due to signal to noise limitations). Despite the non-interconnectivity, the $\mathrm{C}^{18} \mathrm{O}$ hierarchical stratification is consistent with that found with ${ }^{13} \mathrm{CO}$ though with less branching.

The properties of the dendrogram leaves are summarised in Tables A.1 and A.2. The first column indicates the region to which each leaf belongs and the second column shows the dendrogram ID number (cf. Figs. A.1-A.3). The following columns show the properties derived at the lowest contour of each detected leaf: $R$ is the radius; $v_{0}$ is the mean velocity, weighted by the intensity (with an uncertainty of $0.1 \mathrm{~km} \mathrm{~s}^{-1}$ ); $\sigma_{v}$ is the velocity dispersion; $M$ is the leaf mass (Eq. (A.1)); and $\alpha$ is the virial parameter (Eq. (A.2)). Bear in mind that the virial parameters shown in this table are estimated at the bottom of each leaf, but they evolve throughout the height of the leaves, generally approaching subvirial values (Fig. A.1). In addition, the row before-last shows the properties of the branch that covers the entire central region, and the last row shows the total mass, equivalent size and virial parameter of the entire B59. For ${ }^{13} \mathrm{CO}$, the properties of the entire B59 were measured at the root of the dendrogram (root 44, Fig. A.1 left panel), while for $\mathrm{C}^{18} \mathrm{O}$ this was estimated by combining the information of the three disconnected roots (Fig. A.1 right panel, roots 50, 47 and leaf 3).

These estimates do not account for the optical depth, which will particularly affect the central region of B59. Figure 4 quantifies the possible effect of optical depth for $\mathrm{C}^{18} \mathrm{O}$ as deviations at higher column densities above the green dashed line. This would account for a difference of $\sim 15 \%$ on average in the column density in this central region, which translates into an equivalent increase of the mass estimate (and decrease of $\alpha$ ).

\section{A.2.2. The leaves of B59}

The densest gas within the central clump of B59, is solely described by two leafs in both molecular lines, leaves 4 and 6, both gravitationally bound, and both at the systemic velocity of the cloud (in $\mathrm{C}^{18} \mathrm{O}$ ). These correspond to the two cores surrounding 


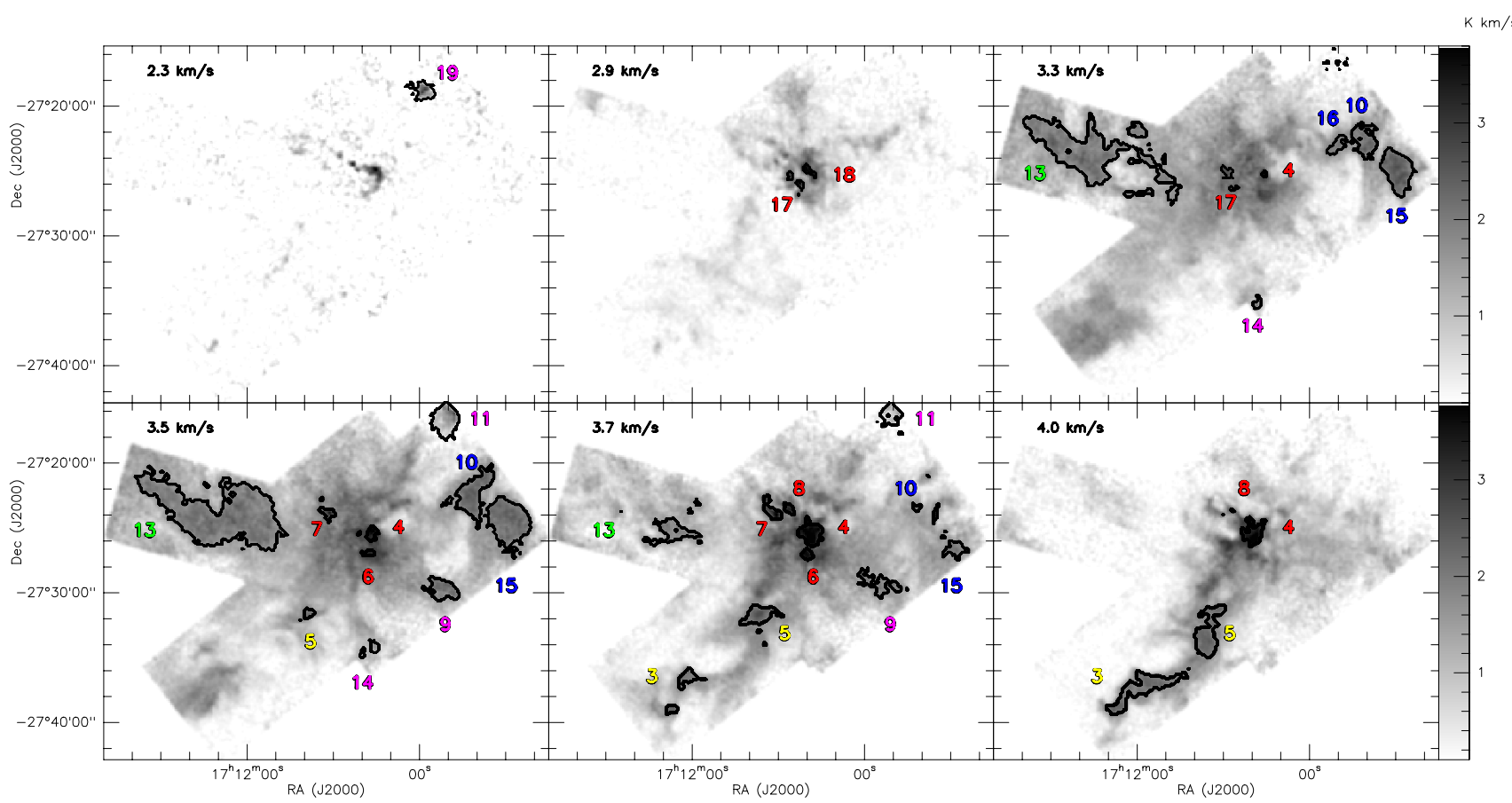

Fig. A.2. Six channel maps of the ${ }^{13} \mathrm{CO}$ emission, $T_{\mathrm{A}}^{*}$, whose velocities are shown at the top-left corner of each panel. The plotted grayscale is that used for all panels with the exception of the first (top-left panel), where it is from 0.1 to $1.5 \mathrm{~K} \mathrm{~km} \mathrm{~s}^{-1}$. The black contours show the positions of the leaves from Fig. A.1, whose numbering is colour-coded to visually separate the distinct areas: central region in red, NE ridge in green, SE U-shaped ridge in yellow, western cores in blue, and loose knots in purple.

Table A.1. Properties of the ${ }^{13} \mathrm{CO}$ dendrogram leaves.

\begin{tabular}{lcccccc}
\hline \hline Region & Leaf \# & $\begin{array}{c}R \\
(\mathrm{pc})\end{array}$ & $\begin{array}{c}v_{0} \\
\left(\mathrm{~km} \mathrm{~s}^{-1}\right)\end{array}$ & $\begin{array}{c}\sigma_{v} \\
\left(\mathrm{~km} \mathrm{~s}^{-1}\right)\end{array}$ & $\begin{array}{c}M \\
\left(M_{\odot}\right)\end{array}$ & $\alpha$ \\
\hline Central & 4 & 0.03 & 3.9 & 0.19 & 0.63 & 2.3 \\
clump & 6 & 0.02 & 3.7 & 0.13 & 0.10 & 3.6 \\
& 18 & 0.02 & 2.9 & 0.13 & 0.10 & 3.7 \\
& 8 & 0.01 & 3.9 & 0.07 & 0.04 & 2.0 \\
& 17 & 0.04 & 3.2 & 0.15 & 0.31 & 3.2 \\
& 7 & 0.03 & 3.7 & 0.11 & 0.19 & 1.9 \\
\hline U-shaped & 5 & 0.06 & 3.9 & 0.14 & 0.57 & 2.5 \\
ridge & 3 & 0.07 & 4.0 & 0.10 & 0.47 & 1.6 \\
\hline NE ridge & 13 & 0.14 & 3.5 & 0.13 & 3.70 & 0.8 \\
\hline Western & 10 & 0.05 & 3.4 & 0.14 & 0.63 & 1.9 \\
cores & 16 & 0.03 & 3.2 & 0.15 & 0.20 & 3.9 \\
& 15 & 0.06 & 3.4 & 0.15 & 1.15 & 1.5 \\
\hline SW lobe & 9 & 0.04 & 3.6 & 0.11 & 0.28 & 1.9 \\
\hline SW lobe & 14 & 0.03 & 3.4 & 0.08 & 0.06 & 3.6 \\
\hline NW lobe & 11 & 0.04 & 3.5 & 0.11 & 0.21 & 2.6 \\
\hline NW lobe & 19 & 0.04 & 2.2 & 0.20 & 0.13 & 13.6 \\
\hline Total centre & $(32)$ & 0.11 & - & 0.33 & 7.87 & 1.9 \\
\hline Total & $(44)$ & 0.44 & - & 0.41 & 73.3 & 1.1 \\
\hline & & & & & &
\end{tabular}

the youngest protostars of the region: $\mathrm{B} 7, \mathrm{~B} 8$ and $\mathrm{B} 11$ within leaf 4 , and B10 within leaf 6 . Apart from these, we have four leaves with ${ }^{13} \mathrm{CO}$ in the central clump (leaves 7, 8, 17 and 18, labeled in red in Fig. A.2). These are a result of the ${ }^{13} \mathrm{CO}$ self absorption and outflow wings, which produce an overly substructured tree tracing outflowing gas at opposing velocities, and artificially separated by the self-absorption dip. These leaves do not have the systemic velocity of $3.5 \mathrm{~km} \mathrm{~s}^{-1}$ but they vary from 2.9 to $3.9 \mathrm{~km} \mathrm{~s}^{-1}$ and trace the dense outflowing gas from Flow 1 .
Table A.2. Properties of the $\mathrm{C}^{18} \mathrm{O}$ dendrogram leaves.

\begin{tabular}{lcccccc}
\hline \hline Region & Leaf \# & $\begin{array}{c}R \\
(\mathrm{pc})\end{array}$ & $\begin{array}{c}V_{0} \\
\left(\mathrm{~km} \mathrm{~s}^{-1}\right)\end{array}$ & $\begin{array}{c}\sigma_{v} \\
\left(\mathrm{~km} \mathrm{~s}^{-1}\right)\end{array}$ & $\begin{array}{c}M \\
\left(M_{\odot}\right)\end{array}$ & $\alpha$ \\
\hline Central & 4 & 0.02 & 3.4 & 0.14 & 0.19 & 1.6 \\
clump & 6 & 0.03 & 3.5 & 0.21 & 1.44 & 1.1 \\
\hline $\begin{array}{l}\text { U-shaped } \\
\text { ridge }\end{array}$ & 3 & 0.06 & 3.4 & 0.13 & 0.91 & 1.5 \\
\hline NE ridge & 13 & 0.15 & 3.5 & 0.15 & 5.82 & 0.7 \\
\hline Western & 15 & 0.07 & 3.4 & 0.15 & 1.51 & 1.2 \\
cores & 10 & 0.07 & 3.3 & 0.21 & 1.15 & 2.9 \\
\hline West centre & 21 & 0.05 & 3.5 & 0.17 & 0.52 & 3.3 \\
\hline Total centre & $(45)$ & 0.10 & - & 0.29 & 16.1 & 0.7 \\
\hline Total & & 0.30 & - & 0.31 & 29.6 & 1.0 \\
\hline
\end{tabular}

The SE U-shaped ridge is divided into two halves in both isotopologues (labeled in yellow in Figs. A.2 and A.3). The lowerleft half of the U-shaped ridge is identified with leaf 3 , at slightly redshifted velocities, consistent with the velocity map of $\mathrm{C}^{18} \mathrm{O}$ (Fig. 9). The other half of the ridge is found as a red-shifted leaf in ${ }^{13} \mathrm{CO}$ (leaf 5), with a high virial parameter, consistent with compressed gas from the redshifted outflow (Flow 2).

The entire NE ridge is found as leaf 13 (labeled in green in Figs. A.2 and A.3), without any sub-structure within it, and at the systemic velocity of the cloud $\left(\sim 3.5 \mathrm{~km} \mathrm{~s}^{-1}\right)$. This is the only leaf in B59 that appears with a $\alpha$ below 1 in both ${ }^{13} \mathrm{CO}$ and $\mathrm{C}^{18} \mathrm{O}$.

On the other hand, the western cores branch out from the main tree and form a sub-structure of their own. They include leaves 10 and 15 (labeled in blue in Figs. A.2 and A.3), detected with both molecules. The mass and virial parameter of leaf 15 , in particular, indicates that it could collapse to form future protostars, but at present it presents no evidence for ongoing star 


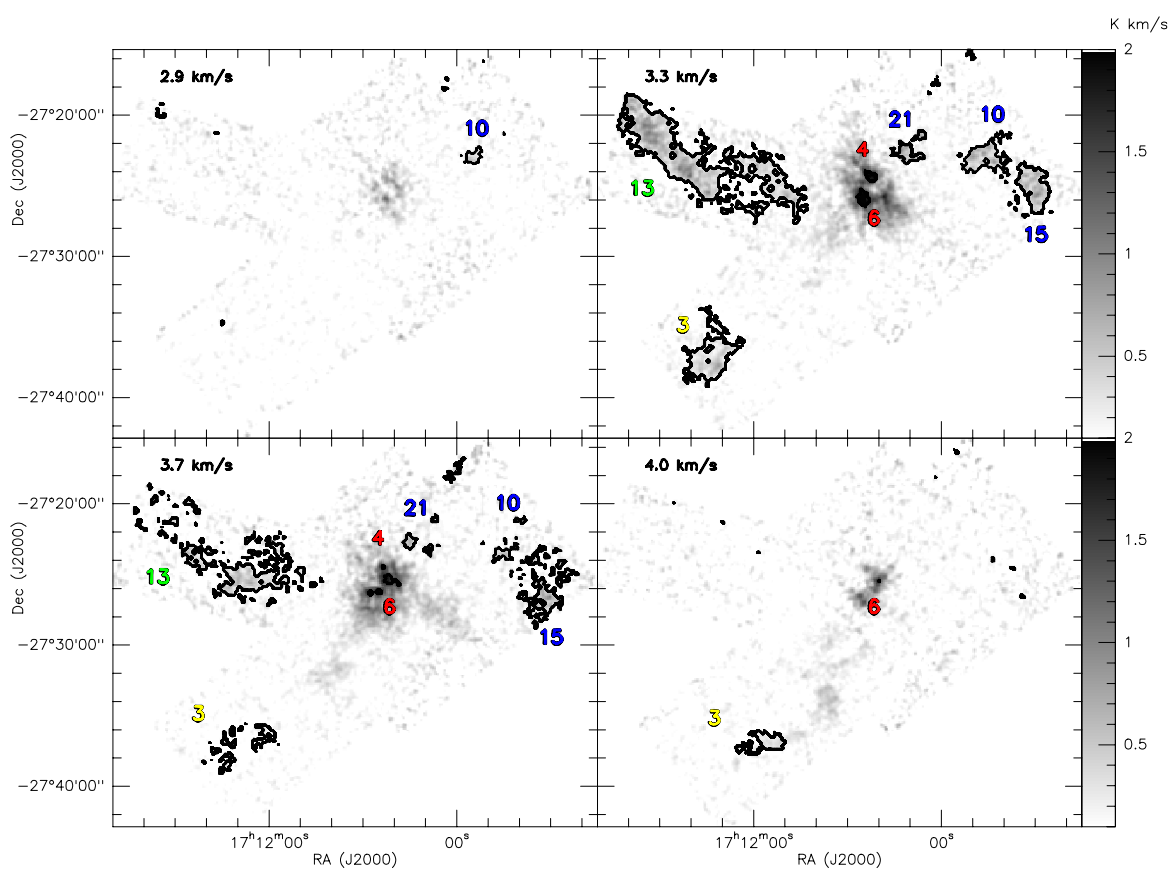

Fig. A.3. Four channel maps of the $\mathrm{C}^{18} \mathrm{O}$ emission in $T_{\mathrm{A}}^{*}$ (in gray scale), whose velocities are shown at the top-left corner of each panel. The black contours show the positions of the leaves from Fig. A.1, and are numbered accordingly. The colour code is the same as for Fig. A.2.

formation. Despite having a similar mass to the central cores of B59, its larger size (and therefore lower densities) are indicative of a less compact core, which has at its disposal a smaller mass reservoir from where it could accrete. If it does become active, it will likely form no more than a single low mass protostar/protostellar system. Spatially nearby, there is the blueshifted ${ }^{13} \mathrm{CO}$ leaf 16 , and the $\mathrm{C}^{18} \mathrm{O}$ leaf 21 , both with a high virial parameter. These two leaves lay at the edges of Cavity C, suggesting that they are part of the material pushed out of the cavity by an outflow. This gas-deprived cavity is now what physically separates the central region and the western cores.

Finally, we hypothesise that the ${ }^{13} \mathrm{CO}$ leaves 9,11 and 14 (labeled in purple in Fig. A.2) are counterparts of the main outflows of the region, but since they are not at very large velocity offsets and since we cannot trace the material between these knots and the respective driving source, it is not straightforward to understand their origin. Leaf 19, in particular, appears isolated and is a possible outflow bullet, with the highest virial parameter and the highest blue shifted velocity in the cloud (at $\sim 2.2 \mathrm{~km} \mathrm{~s}^{-1}$ ).

\section{A.2.3. The masses}

A brief comparison of the masses derived from the ${ }^{13} \mathrm{CO}$ and $\mathrm{C}^{18} \mathrm{O}$ emission shows that the structures which are common have similar mass estimates. $\mathrm{C}^{18} \mathrm{O}$ recovers more mass from the NE ridge and from some of the cores, as expected from the higher optical depth of ${ }^{13} \mathrm{CO}$. Nevertheless, both lines show a total of $\sim 10 M_{\odot}$ in the leaves. However, they differ in the total mass of the cluster by a factor of $\sim 2.5\left(\sim 74 M_{\odot}\right.$ in ${ }^{13} \mathrm{CO}$ versus $\sim 32 M_{\odot}$ in $\mathrm{C}^{18} \mathrm{O}$ ). Such a difference is mostly due to the diffuse gas traced only by ${ }^{13} \mathrm{CO}$. That said, the fraction of mass in the leaves is $\sim 11 \%$ using the ${ }^{13} \mathrm{CO}$, and $\sim 33 \%$ for the $\mathrm{C}^{18} \mathrm{O}$ estimate.

Considering only the central clump of B59, our best mass estimate is retrieved from $\mathrm{C}^{18} \mathrm{O}$, as estimated at the lower level of branch 45 . This central region is found to have a mass of $\sim 16 M_{\odot}$ within a region of $\sim 0.1 \mathrm{pc}$ radius, with an average velocity dispersion $\left(\sigma_{v}\right)$ of $0.3 \mathrm{~km} \mathrm{~s}^{-1}$ (i.e. $\alpha=0.7$ ).
When using the momentum maps constructed over the entire velocity range, we measure within a central region of $\sim 0.1 \mathrm{pc}$ radii a mass of $10 M_{\odot}$, a mean velocity of $3.5 \mathrm{~km} \mathrm{~s}^{-1}$ and a mean $\sigma_{v}$ of $0.35 \mathrm{~km} \mathrm{~s}^{-1}$. Assuming the uniform profile as that taken for the dendrogram analysis, we calculate a virial parameter of 1.5. The smaller mass estimated using the total integrated intensity is not surprising since we are now taking a circular area around the central region of $0.1 \mathrm{pc}$ radii, out of which not all pixels are included in the calculation due to signal to noise limitations. For the same reason, the higher velocity dispersion is also compatible with the dendrograms, likely arising from the fact that the larger line widths in B59 are concentrated toward the central region. This is also clear looking at the variations of the virial parameter of branch 45 (Fig. A.2, right), which starts at $\sim 2$ at higher levels, decreasing down to 0.7 at the lower level of the branch.

\section{References}

Alves, F. O., \& Franco, G. A. P. 2007, A\&A, 470, 597

Alves, F. O., Franco, G. A. P., \& Girart, J. M. 2008, A\&A, 486, L13

Arce, H. G., \& Sargent, A. I. 2006, ApJ, 646, 1070

Arce, H. G., Borkin, M. A., Goodman, A. A., Pineda, J. E., \& Halle, M. W. 2010, ApJ, 715, 1170

Bohlin, R. C., Savage, B. D., \& Drake, J. F. 1978, ApJ, 224, 132

Bontemps, S., Andre, P., Terebey, S., \& Cabrit, S. 1996, A\&A, 311, 858

Brooke, T. Y., Huard, T. L., Bourke, T. L., et al. 2007, ApJ, 655, 364

Buckle, J. V., Hills, R. E., Smith, H., et al. 2009, MNRAS, 399, 1026

Cabrit, S., \& Bertout, C. 1990, ApJ, 348, 530

Cabrit, S., \& Bertout, C. 1992, A\&A, 261, 274

Carroll, J. J., Frank, A., Blackman, E. G., Cunningham, A. J., \& Quillen, A. C. 2009, ApJ, 695, 1376

Carroll, J. J., Frank, A., \& Blackman, E. G. 2010, ApJ, 722, 145

Cavanagh, B., Jenness, T., Economou, F., \& Currie, M. J. 2008, Astron. Nachr., 329, 295

Covey, K. R., Lada, C. J., Román-Zúñiga, C., et al. 2010, ApJ, 722, 971

Cunningham, A. J., Frank, A., \& Blackman, E. G. 2006, ApJ, 646, 1059

Curtis, E. I., Richer, J. S., \& Buckle, J. V. 2010a, MNRAS, 401, 455

Curtis, E. I., Richer, J. S., Swift, J. J., \& Williams, J. P. 2010b, MNRAS, 408 , 1516

Duarte-Cabral, A., Fuller, G. A., Peretto, N., et al. 2010, A\&A, 519, A27 
Duarte-Cabral, A., Dobbs, C. L., Peretto, N., \& Fuller, G. A. 2011, A\&A, 528, A50

Forbrich, J., Lada, C. J., Muench, A. A., Alves, J., \& Lombardi, M. 2009, ApJ, 704, 292

Forbrich, J., Posselt, B., Covey, K. R., \& Lada, C. J. 2010, ApJ, 719, 691

Frau, P., Girart, J. M., Beltrán, M. T., et al. 2010, ApJ, 723, 1665

Frau, P., Girart, J. M., \& Beltrán, M. T. 2012, A\&A, 537, L9

Frerking, M. A., Langer, W. D., \& Wilson, R. W. 1982, ApJ, 262, 590

Fuller, G. A., \& Ladd, E. F. 2002, ApJ, 573, 699

Hatchell, J., Fuller, G. A., \& Ladd, E. F. 1999, A\&A, 344, 687

Heitsch, F., Ballesteros-Paredes, J., \& Hartmann, L. 2009, ApJ, 704, 1735

Hennebelle, P., \& Chabrier, G. 2011, in IAU Symp. 270, eds. J. Alves, B. G. Elmegreen, J. M. Girart, \& V. Trimble, 159

Ladd, E. F., Fuller, G. A., \& Deane, J. R. 1998, ApJ, 495, 871

Langer, W. D., \& Penzias, A. A. 1993, ApJ, 408, 539

Lombardi, M., Alves, J., \& Lada, C. J. 2006, A\&A, 454, 781

Matzner, C. D. 2007, ApJ, 659, 1394

Matzner, C. D., \& McKee, C. F. 2000, ApJ, 545, 364

Maury, A. J., André, P., \& Li, Z.-Y. 2009, A\&A, 499, 175

McKee, C. F., \& Ostriker, E. C. 2007, ARA\&A, 45, 565

Muench, A. A., Lada, C. J., Rathborne, J. M., Alves, J. F., \& Lombardi, M. 2007, ApJ, 671, 1820
Nakamura, F., \& Li, Z.-Y. 2007, ApJ, 662, 395

Nakamura, F., \& Li, Z. 2008, ApJ, 687, 354

Nakamura, F., Sugitani, K., Shimajiri, Y., et al. 2011, ApJ, 737, 56

Onishi, T., Kawamura, A., Abe, R., et al. 1999, PASJ, 51, 871

Peretto, N., André, P., Könyves, V., et al. 2012, A\&A, 541, A63

Pineda, J. E., Caselli, P., \& Goodman, A. A. 2008, ApJ, 679, 481

Rathborne, J. M., Lada, C. J., Muench, A. A., Alves, J. F., \& Lombardi, M. 2008, ApJS, 174, 396

Riaz, B., Martín, E. L., Bouy, H., \& Tata, R. 2009, ApJ, 700, 1541

Román-Zúñiga, C. G., Lada, C. J., \& Alves, J. F. 2009, ApJ, 704, 183

Román-Zúñiga, C. G., Alves, J. F., Lada, C. J., \& Lombardi, M. 2010, ApJ, 725,

$$
2232
$$

Román-Zúñiga, C. G., Frau, P., Girart, J. M., \& Alves, J. F. 2012, ApJ, 747, 149 Rosolowsky, E. W., Pineda, J. E., Kauffmann, J., \& Goodman, A. A. 2008, ApJ, 679,1338

Rybicki, G. B., \& Lightman, A. P. 1986, Radiative Processes in Astrophysics

Schneider, N., Csengeri, T., Bontemps, S., et al. 2010, A\&A, 520, A49

van der Tak, F. F. S., Black, J. H., Schöier, F. L., Jansen, D. J., \& van Dishoeck, E. F. 2007 , A\&A, 468, 627

Vázquez-Semadeni, E. 2010, in ASP Conf. Ser. 438, eds. R. Kothes, T. L. Landecker, \& A. G. Willis, 83

Vázquez-Semadeni, E., Gómez, G. C., Jappsen, A. K., et al. 2007, ApJ, 657, 870 